\title{
A CAUCHY-KOWALEVSKY THEOREM FOR OVERDETERMINED SYSTEMS OF NONLINEAR PARTIAL DIFFERENTIAL EQUATIONS AND GEOMETRIC APPLICATIONS
}

\author{
M. S. BAOUENDI, P. EBENFELT, D. ZAITSEV
}

\section{Contents}

1. Introduction

2. Notation and conventions

3. Solvability and compatibility conditions

4. Approximate solvability with respect to a slope

5. Exterior differential systems and integral manifolds 12

6. Kähler ordinary and Kähler regular integral elements 16

7. Proof of (ii) $\Longrightarrow$ (i) in Theorem $1.1 \quad 19$

8. A system of Monge-Ampère type 20

9. Proof of Theorem 1.6 25

10. Proof of Proposition 1.7 26

References $\quad 28$

\section{INTRODUCTION}

The main motivation for the work presented in this paper is to construct real hypersurfaces in $\mathbb{C}^{n+1}$ with maximal Levi number (see below for the definition), a problem that has been open since Levi numbers were introduced in BHR96. The examples constructed here are tube hypersurfaces (i.e. of the form $M=\Sigma+i \mathbb{R}^{n+1}$ where $\Sigma$ is a hypersurface in $\mathbb{R}^{n+1}$ ). Moreover, we give a local description of all such hypersurfaces. In order for the real hypersurface $M$ to have the desired properties, $\Sigma$ must be a non-cylindrical hypersurface whose Gauss map (or equivalently second fundamental form) has rank one. To construct locally defined hypersurfaces in $\mathbb{R}^{n+1}$ with these properties, indeed to parametrize all such, we prove an existence and uniqueness theorem concerning a Cauchy problem for a class of overdetermined systems of nonlinear partial differential equations in $\mathbb{R}^{n}$. The Cauchy data is posed on a noncharacteristic $k$-dimensional plane in $\mathbb{R}^{n}$, where

\footnotetext{
${ }^{0}$ The first two authors are supported in part by DMS-0701070 and DMS-0701121. The third author is supported in part by the Science Foundation Ireland.
} 
the dimension $k$ depends, roughly, on how overdetermined the system is. In the special case where the system is not overdetermined at all, we have $k=n-1$ and our theorem reduces to the classical Cauchy-Kowalevsky theorem.

Consider the Cauchy problem for an unknown vector-valued function $u:=\left(u^{1}, \ldots, u^{m}\right)$ given by

$$
\left\{\begin{array}{l}
\frac{\partial u^{A}}{\partial x^{\alpha}}=F_{\alpha}^{A}\left(x, u, \frac{\partial u}{\partial x^{1}}, \ldots, \frac{\partial u}{\partial x^{k}}\right) \\
u^{A}\left(x^{1} \ldots, x^{k}, x_{0}^{k+1}, \ldots, x_{0}^{n}\right)=a^{A}\left(x^{1}, \ldots, x^{k}\right)
\end{array}, \quad A=1, \ldots, m, \alpha=k+1, \ldots, n\right.
$$

where $F_{\alpha}^{A}\left(x, p, p^{\prime}\right)$ are real-valued, real-analytic functions in an open subset $U$ of $\mathbb{R}^{n} \times \mathbb{R}^{m} \times$ $\mathbb{R}^{m k},\left(x_{0}, p_{0}, p_{0}^{\prime}\right)$ a fixed point in $U$, and the Cauchy data $a=\left(a^{1}, \ldots a^{m}\right)$ are real-analytic in a neighborhood of $\left(x_{0}^{1}, \ldots, x_{0}^{k}\right)$ in $\mathbb{R}^{k}$ with

$$
\left(a\left(x_{0}^{1}, \ldots, x_{0}^{k}\right),\left(\frac{\partial a}{\partial x^{1}}\left(x_{0}^{1}, \ldots, x_{0}^{k}\right), \ldots, \frac{\partial a}{\partial x^{k}}\left(x_{0}^{1}, \ldots, x_{0}^{k}\right)\right)\right)=\left(p_{0}, p_{0}^{\prime}\right) .
$$

We shall say that the Cauchy problem (1.1) is solvable at $\left(x_{0}, p_{0}, p_{0}^{\prime}\right)$ for every initial data if there is a unique real-analytic solution $u(x)$ to (1.1) in an open neighborhood of $x_{0}$ for every Cauchy data $a\left(x^{1}, \ldots x^{k}\right)$ satisfying (1.2).

In order to formulate our main result, we need to introduce one more notion. We shall say that a real-analytic $\mathbb{R}^{m}$-valued function $u(x)$ defined in an open neighborhood of $x_{0}$ is an approximate solution at $x_{0}$ to the Cauchy problem (1.1) if

$$
\left\{\begin{array}{l}
\frac{\partial u^{A}}{\partial x^{\alpha}}=F_{\alpha}^{A}\left(x, u, \frac{\partial u}{\partial x^{1}}, \ldots, \frac{\partial u}{\partial x^{k}}\right)+O\left(\sum_{i=1}^{n}\left|x^{i}-x_{0}^{i}\right|^{2}\right), \\
u^{A}\left(x^{1} \ldots, x^{k}, x_{0}^{k+1}, \ldots, x_{0}^{n}\right)=a^{A}\left(x^{1}, \ldots, x^{k}\right)+O\left(\sum_{j=1}^{k}\left|x^{j}-x_{0}^{j}\right|^{3}\right),
\end{array}\right.
$$

where $A$ and $\alpha$ are as in (1.1). Note that the property of $u(x)$ being an approximate solution at $x_{0}$ to the system (1.1) only depends on its 2-jet at $x_{0}$ as well as the 2-jet of the initial data $a\left(x^{1}, \ldots, x^{k}\right)$ at $\left(x_{0}^{1}, \ldots, x_{0}^{k}\right)$. The Cauchy problem (1.1) is said to be approximately solvable for every initial data at $\left(x_{0}, p_{0}, p_{0}^{\prime}\right) \in U$ if for every initial data a defined in a neighborhood of $\left(x_{0}^{1}, \ldots, x_{0}^{k}\right)$ and satisfying (1.2), there is an approximate solution $u$ to (1.1) at $x_{0}$. Our main result is the following.

Theorem 1.1. Let $F_{\alpha}^{A}=F_{\alpha}^{A}\left(x, p, p^{\prime}\right)$, for $A=1, \ldots, m$ and $\alpha=k+1, \ldots, n$, be realvalued, real-analytic functions defined in an open subset $U$ of $\mathbb{R}^{n} \times \mathbb{R}^{m} \times \mathbb{R}^{m k}$. The following are equivalent.

(i) The Cauchy problem (1.1) is solvable for every initial data at every $\left(x_{0}, p_{0}, p_{0}^{\prime}\right) \in U$.

(ii) The Cauchy problem (1.1) is approximately solvable for every initial data at every $\left(x_{0}, p_{0}, p_{0}^{\prime}\right) \in U$. 
(iii) The functions $F_{\alpha}^{A}$ satisfy the compatibility conditions

$$
\begin{aligned}
\Phi_{\alpha \beta}^{A}\left(x, p, p^{\prime}\right) & =\Phi_{\beta \alpha}^{A}\left(x, p, p^{\prime}\right), \\
\Psi_{\alpha \beta C}^{A \Gamma \Lambda}\left(x, p, p^{\prime}\right) & =\Psi_{\beta \alpha C}^{A \Gamma \Lambda}\left(x, p, p^{\prime}\right),
\end{aligned}
$$

for all $A, C=1, \ldots, m, \Lambda, \Gamma=1, \ldots, k, \alpha, \beta=k+1, \ldots, n$, and $\left(x, p, p^{\prime}\right) \in U$, where

$$
\begin{aligned}
\Phi_{\alpha \beta}^{A} & :=\frac{\partial F_{\alpha}^{A}}{\partial x^{\beta}}+\sum_{B=1}^{m} \frac{\partial F_{\alpha}^{A}}{\partial p^{B}} F_{\beta}^{B}+\sum_{\Gamma=1}^{k} \sum_{B=1}^{m} \frac{\partial F_{\alpha}^{A}}{\partial p_{\Gamma}^{B}}\left(\frac{\partial F_{\beta}^{B}}{\partial x^{\Gamma}}+\sum_{C=1}^{m} \frac{\partial F_{\beta}^{B}}{\partial p^{C}} p_{\Gamma}^{C}\right), \\
\Psi_{\alpha \beta C}^{A \Gamma \Lambda} & :=\sum_{B=1}^{m}\left(\frac{\partial F_{\alpha}^{A}}{\partial p_{\Gamma}^{B}} \frac{\partial F_{\beta}^{B}}{\partial p_{\Lambda}^{C}}+\frac{\partial F_{\alpha}^{A}}{\partial p_{\Lambda}^{B}} \frac{\partial F_{\beta}^{B}}{\partial p_{\Gamma}^{C}}\right)
\end{aligned}
$$

with the notation

$$
p=\left(p^{A}\right)^{A=1, \ldots, m}, \quad p^{\prime}=\left(p_{\Gamma}^{A}\right)_{\Gamma=1, \ldots, k}^{A=1, \ldots, m} .
$$

As an illustration of Theorem 1.1, we give an example where the conditions (1.4) and (1.5) take a particularly simple form.

Example 1.2. Let $L_{\alpha B}^{A}$, for $A, B=1, \ldots, m$ and $\alpha=k+1, \ldots, n$, be vector fields of the form

$$
L_{\alpha B}^{A}=\delta_{B}^{A} \frac{\partial}{\partial x^{\alpha}}+\sum_{\Lambda=1}^{k} \xi_{\alpha B}^{A \Lambda}(x) \frac{\partial}{\partial x^{\Lambda}}
$$

where $\delta_{B}^{A}$ denotes the Kronecker delta and the coefficients $\xi_{\alpha B}^{A \Lambda}(x)$ are real-valued and real-analytic in an open subset $\Omega$ of $\mathbb{R}^{n}$. Let $\mathcal{L}_{\alpha}$ be the $m \times m$ matrix of vector fields $\mathcal{L}_{\alpha}:=\left(L_{\alpha B}^{A}\right)_{A, B=1, \ldots m}$ acting on $\mathbb{R}^{m}$-valued functions $u=\left(u^{B}\right)_{B=1, \ldots, m}$ by

$$
\mathcal{L}_{\alpha} u:=\left(\sum_{B=1}^{m} L_{\alpha B}^{A} u^{B}\right)_{A=1, \ldots, m} .
$$

Clearly, the Cauchy problem for $u=\left(u^{A}\right)_{A=1, \ldots, m}$ given by

$$
\left\{\begin{array}{l}
\mathcal{L}_{\alpha} u=0 \\
u^{A}\left(x^{1} \ldots, x^{k}, x_{0}^{k+1}, \ldots, x_{0}^{n}\right)=a^{A}\left(x^{1}, \ldots, x^{k}\right)
\end{array}, \quad A=1, \ldots, m, \alpha=k+1, \ldots, n,\right.
$$

where $x^{0} \in \Omega$, can be written in the form (1.1). An elementary computation shows that the compatibility conditions (1.4) and (1.5) are equivalent to the generalized Frobenius condition

$$
\left[\mathcal{L}_{\alpha}, \mathcal{L}_{\beta}\right]:=\mathcal{L}_{\alpha} \mathcal{L}_{\beta}-\mathcal{L}_{\beta} \mathcal{L}_{\alpha}=0
$$

Note that $\mathcal{L}_{\alpha} \mathcal{L}_{\beta}$ is an $m \times m$ matrix of second order differential operators and the commutator $\left[\mathcal{L}_{\alpha}, \mathcal{L}_{\beta}\right]$ is, in general, also an $m \times m$ matrix of second order operators. If $m=1$, then the commutator $\left[\mathcal{L}_{\alpha}, \mathcal{L}_{\beta}\right]$ is a vector field, and Theorem 1.1 for the Cauchy problem (1.8) reduces to the standard Frobenius theorem (in the real-analytic category). 
As a first step in the proof of Theorem 1.1, we shall prove (i) $\Longrightarrow$ (iii) (i.e. the necessity of the compatibility conditions) and (ii) $\Longleftrightarrow$ (iii) (i.e. the characterization of the compatibility conditions by means of approximate solutions). This will be done in Section 3. To complete the proof, we show, in Section 17, that (ii) $\Longrightarrow$ (i). This step is more delicate and uses Cartan-Kähler theory (see e.g. [BCG $\left.{ }^{3} 91\right]$ ). In Sections 4, 5, and 6. we introduce the necessary preliminary material for the proof of (ii) $\Longrightarrow$ (i).

The study of nonlinear overdetermined systems of analytic partial differential equations has a long history going back to the late 19th century. We mention here only some this work, namely that of E. Cartan [C31], C. Riquier [R10], J. M. Thomas [T34], and H. Goldschmidt G67] in which results on local and formal existence of solutions to the systems are given. The interested reader is referred to [BCG $\left.{ }^{3} 91\right]$ for further references.

We shall apply Theorem 1.1 to a Cauchy problem for a system of Monge-Ampère type equations that will be used for the geometric applications mentioned above. Let $x=\left(x^{1}, \ldots, x^{n}\right)$ denote coordinates in $\mathbb{R}^{n}$. For a function $u=u(x)$, we shall use the notation

$$
u_{i}:=u_{x^{i}}=\partial_{x^{i}} u=\frac{\partial u}{\partial x^{i}}, \quad u_{i j}:=u_{x^{i} x^{j}}=\partial_{x^{i}} \partial_{x^{j}} u=\frac{\partial^{2} u}{\partial x^{i} \partial x^{j}} .
$$

For integers $2 \leq \alpha, \beta \leq n$, we shall denote by $\Delta_{\alpha \beta}$ the second order, nonlinear partial differential operator

$$
\Delta_{\alpha \beta}(u):=\operatorname{det}\left(\begin{array}{cc}
u_{11} & u_{1 \beta} \\
u_{\alpha 1} & u_{\alpha \beta}
\end{array}\right) .
$$

We have the following result, whose proof is given in Section 8 as an application of Theorem 1.1.

Theorem 1.3. Let $n>2$ and $f_{\alpha \beta}(x, t), 2 \leq \alpha, \beta \leq n$, be real-analytic, real-valued functions in a connected open subset $U \times V \subset \mathbb{R}^{n} \times(\overline{\mathbb{R}} \backslash\{0\})$. Then the following two conditions are equivalent:

(i) For any $x_{0}=\left(x_{0}^{1}, \ldots, x_{0}^{n}\right) \in U$ and any real-valued, real-analytic functions a $\left(x^{1}\right)$, $a_{2}\left(x^{1}\right), \ldots, a_{n}\left(x^{1}\right)$ in a neighborhood of $x_{0}^{1}$ in $\mathbb{R}$ such that $a^{\prime \prime}\left(x_{0}^{1}\right) \in V$, there exists a unique real-valued, real-analytic solution $u(x)$ in a neighborhood of $x_{0}$ to the Cauchy problem

$$
\left\{\begin{array}{l}
\Delta_{\alpha \beta}(u)=f_{\alpha \beta}\left(x, u_{11}\right), \\
u\left(x^{1}, x_{0}^{2}, \ldots, x_{0}^{n}\right)=a\left(x^{1}\right), \quad u_{\alpha}\left(x^{1}, x_{0}^{2}, \ldots, x_{0}^{n}\right)=a_{\alpha}\left(x^{1}\right), \quad \alpha, \beta=2, \ldots, n .
\end{array}\right.
$$

(ii) The functions $f_{\alpha \beta}$ are of the form $f_{\alpha \beta}(x, t)=g_{\alpha \beta}(x) t$ with $g_{\alpha \beta}$ satisfying the compatibility conditions

$$
g_{\alpha \beta}=g_{\beta \alpha}, \quad \partial_{x^{1}} g_{\alpha \beta}=0, \quad \partial_{x^{\gamma}} g_{\alpha \beta}=\partial_{x^{\beta}} g_{\alpha \gamma}, \quad \alpha, \beta, \gamma=2, \ldots, n .
$$

Remark 1.4. The conditions (1.11) are locally equivalent to the existence of a realanalytic function $v(x)$ independent of $x_{1}$ with $g_{\alpha \beta}=\partial_{x_{\alpha}} \partial_{x_{\beta}} v$, for $2 \leq \alpha, \beta \leq n$. Also 
observe that if $f_{\alpha \beta}(x, t)$ are independent of $t$, then (ii) is equivalent to the vanishing of all $f_{\alpha \beta}$.

Remark 1.5. Observe that when $n=2$, the condition (i) of Theorem 1.3 holds for any right hand side $f_{\alpha \beta}=f_{22}$ as a consequence of the Cauchy-Kowalevsky Theorem. Hence, the implication (i) $\Longrightarrow$ (ii) does not hold for $n=2$.

Next, recall that if $\Sigma$ is a smooth oriented hypersurface in $\mathbb{R}^{n+1}$, then its Gauss map $G: \Sigma \rightarrow S^{n}$ is the function that sends $x \in \Sigma$ to the (positive) unit normal $n(x) \in S^{n}$. The Gauss (or spherical) image of $\Sigma$ is $G(\Sigma) \subset S^{n}$. If $\Sigma$ is (locally) defined in $\mathbb{R}^{n+1}$ as a graph $y=u(x)$, then the rank of the Gauss map at a point $(x, u(x)) \in \mathbb{R}^{n} \times \mathbb{R}$ is precisely the rank of the Hessian of $u$ at $x$. Consequently, the graphs $y=u(x)$ for which the rank of the Gauss map is identically one (with the additional harmless assumption that $u_{11} \neq 0$ ) correspond precisely to the solutions of the system of equations $\Delta_{\alpha \beta}(u)=0$, $\alpha, \beta=2, \ldots, n$. The Cauchy data $a\left(x^{1}\right), a_{\alpha}\left(x^{1}\right), 2 \leq \alpha \leq n$, in (1.10) can be used to prescribe the Gauss image of $\Sigma$. More precisely, we have the following result, whose proof is given in Section 9 as an application of Theorem 1.3 .

Theorem 1.6. Let $\gamma:(-1,1) \rightarrow S^{n}$ be a real-analytic curve with $\gamma(0)=(0, \ldots, 0,-1)$ and $\gamma^{\prime}(0)=(1,0 \ldots, 0)$. Then, there exists a unique (germ at 0 of a) real-analytic function $u(x), x \in \mathbb{R}^{n}$, with $u(0)=0$ such that if $\Sigma \subset \mathbb{R}^{n+1}$ is the graph of $u(x)$ and $G$ is its Gauss map, then the rank of $G$ is equal to one at every point of $\Sigma$ and $G\left(\left(x^{1}, 0\right), u\left(x^{1}, 0\right)\right)=\gamma\left(x^{1}\right)$.

We remark that this local result contrasts with the global situation: Hartman and Nirenberg [HN59] showed that any properly embedded smooth, connected hypersurface in $\mathbb{R}^{n+1}$ whose Gauss map has rank $\leq 1$ at every point (or, equivalently, whose Riemannian curvature tensor vanishes identically at every point) is necessarily a cylinder over a curve, and hence its Gauss image is contained in a 2-plane section of the sphere, i.e. is contained in a circle. This strong global rigidity fails for hypersurfaces whose Gauss map has rank two or higher. There are relatively simple examples of properly embedded real-analytic hypersurfaces in $\mathbb{R}^{n+1}$ whose Gauss maps have rank two, but whose Gauss images are not even contained in any hyperplane section of the sphere (see [S60] and [W95]; see also AG04]).

To describe our result concerning real hypersurfaces in $\mathbb{C}^{n+1}$, we need some definitions. Let $M$ be a real-analytic, connected hypersursurface in $\mathbb{C}^{n+1}$. Recall that $M$ is said to be holomorphically nondegenerate if there are no nontrivial germs of holomorphic vector fields, i.e. holomorphic sections of $T^{1,0} \mathbb{C}^{n+1}$, that are tangent to $M$. If $p \in M$, we let $\rho(Z, \bar{Z})$ be a local defining function for $M$ near $p$. We let $L_{1}, \ldots, L_{n}$ be a local basis for the CR vector fields on $M$ near $p$. The hypersurface $M$ is said to be $k$-nondegenerate at $p$ if the collection of vectors

$$
L^{I} \rho_{Z}(p, \bar{p}), \quad I \in \mathbb{Z}_{+}^{n},|I| \leq j,
$$

spans $\mathbb{C}^{n+1}$ for $j=k$ and $k$ is the smallest integer with this property; here, we use standard multi-index notation $L^{I}:=L_{1}^{I_{1}} \ldots L_{n}^{I_{n}}$, etc. The notion of $k$-nondegeneracy is 
independent of the choice of defining function $\rho$ and $\mathrm{CR}$ vector fields $L_{1}, \ldots, L_{n}$. We shall say that $M$ is finitely nondegenerate at $p$ if it is $k$-nondegenerate at $p$ for some $k$. The reader is referred to BER99] for further information about these notions and CR geometry in general. We mention that $M$ is holomorphically nondegenerate if and only if $M$ is finitely nondegenerate on a dense open subset. For a holomorphically nondegenerate hypersurface $M$, its Levi number, denoted $\ell(M)$, is defined to be the minimal $k$ such that $M$ is $k$-nondegenerate at some point (and hence for all points in a dense open subset). As mentioned above, the Levi number was introduced in [BHR96], where it was also shown that $\ell(M)$ is always $\leq n$. If $M$ is Levi nondegenerate at some point, then $\ell(M)=1$ and, hence, real hypersurfaces in general position satisfy $\ell(M)=1$. We remark that it is trivial to construct, for any integer $k$, a real-analytic hypersurface $M$ that is $k$-nondegenerate at a given point $p$. However, the Levi number of such an $M$ will still be, generically, equal to one. Examples of hypersurfaces $M$ with $\ell(M) \geq 2$ are scarce. The first example of an $M$ in $\mathbb{C}^{3}$ with $\ell(M)=2$ can be found in [F77]. Another example with $\ell(M)=2$ is the tube over the light cone. Homogeneous tube hypersurfaces in $\mathbb{C}^{3}$ with Levi number $=2$ were systematically studied in [FK06], [FK07]. An example in $\mathbb{C}^{4}$ with Levi number $=3$ was constructed in [F07]. To the best of the authors' knowledge, no examples with Levi number $\geq 4$ are known in the literature. In this paper we show that, for any $n \geq 1$, there are (plenty of) real-analytic tube hypersurfaces in $\mathbb{C}^{n+1}$ with Levi number $=n$. This statement is an immediate consequence of Theorem 1.6 and the following proposition; see also Remark 10.4.

Proposition 1.7. Let $\Sigma$ be a real-analytic, connected hypersurface in $\mathbb{R}^{n+1}$. Assume that its Gauss image $G(\Sigma)$ is a real-analytic curve in $S^{n} \subset \mathbb{R}^{n+1}$ that is not contained in a hyperplane. Then, the real-analytic tube hypersurface $M:=\Sigma+i \mathbb{R}^{n+1} \subset \mathbb{C}^{n+1}$ is holomorphically nondegenerate, the rank of the Levi form of $M$ is one on a dense open subset of $M$, and the Levi number $\ell(M)=n$.

Finally, we remark that a holomorphically nondegenerate tube hypersurface $M:=$ $\Sigma+i \mathbb{R}^{n+1}$ in $\mathbb{C}^{n+1}$ such that the rank of its Levi form is one at every point, the existence of which is guaranteed by Theorem 1.6 and Proposition 1.7, is only locally defined, i.e. not properly embedded in $\mathbb{C}^{n+1}$. This follows again from the theorem of Hartman and Nirenberg: If $M$ were properly embedded, then $\Sigma$ would be a properly embedded, noncylindrical hypersurface whose Gauss map has rank one at every point, contradicting the theorem of Hartman and Nirenberg.

This paper is organized as follows. In Section 2, we introduce some basic notation and conventions that will be used throughout the paper. The general Cauchy problem and the main result, Theorem 1.1, concerning the existence and uniqueness of solutions are formulated. The proof of this theorem is then given in Sections 13, 4, 5, 6, and 7, The proof of Theorem 1.3 is given in Section 8. The final two sections are devoted to the proofs of Theorem 1.6 and Proposition 1.7, respectively. 
The authors wish to thank Robert Bryant for comments on an earlier version of this paper, and in particular for providing us with relevant references.

\section{NotATiOn AND CONVENTIONS}

In this section, we shall introduce some notation and conventions that will be used in this paper. Let $1 \leq k<n$ be integers and $x=\left(x^{1}, \ldots, x^{k}, x^{k+1}, \ldots, x^{n}\right)$ denote coordinates in $\mathbb{R}^{n}=\mathbb{R}^{k} \times \mathbb{R}^{n-k}$. We shall use the following conventions. Small Latin letters $i, j$, etc. will run over the set $\{1, \ldots, n\}$, capital Greek letters $\Lambda, \Gamma$, etc. will run over $\{1, \ldots, k\}$, small Greek letters $\alpha, \beta$, etc. will run over $\{k+1, \ldots, n\}$, and capital Latin letters $A, B$, etc. will run over $\{1, \ldots, m\}$. Thus, we shall refer to the coordinates as $x^{i}$, and $x^{\Lambda}$ can be used as coordinates on the initial $k$-plane $\left\{x^{\alpha}=x_{0}^{\alpha}\right\}$ in the Cauchy problem (1.1). The unknown function will be denoted $u^{A}$. We shall use the notation

$$
u_{i}^{A}:=\frac{\partial u^{A}}{\partial x^{i}}
$$

The right hand side of the system of partial differential equations in (1.1) will be denoted by $F_{\alpha}^{A}\left(x^{i}, p^{B}, p_{\Lambda}^{B}\right)$ with the notation introduced in (1.7). The Cauchy problem (1.1) can now be written as

$$
\left\{\begin{array}{l}
u_{\alpha}^{A}=F_{\alpha}^{A}\left(x^{i}, u^{B}, u_{\Gamma}^{B}\right) \\
u^{A}\left(x^{\Lambda}, x_{0}^{\alpha}\right)=a^{A}\left(x^{\Lambda}\right)
\end{array}, \quad A=1, \ldots, m, \alpha=k+1, \ldots, n,\right.
$$

the point $\left(x_{0}, p_{0}, p_{0}^{\prime}\right)$ as $\left(\left(x^{i}\right)_{0},\left(p^{A}\right)_{0},\left(p_{\Lambda}^{A}\right)_{0}\right)$, and the condition (1.2) as

$$
\left(a^{A}\left(x_{0}^{\Gamma}\right), a_{\Lambda}^{A}\left(x_{0}^{\Gamma}\right)\right)=\left(\left(p^{A}\right)_{0},\left(p_{\Lambda}^{A}\right)_{0}\right) .
$$

We shall use the following notation for the derivatives of $F_{\alpha}^{A}$

$$
F_{\alpha i}^{A}:=\frac{\partial F_{\alpha}^{A}}{\partial x^{i}}, \quad F_{\alpha B}^{A}:=\frac{\partial F_{\alpha}^{A}}{\partial p^{B}}, \quad F_{\alpha B}^{A \Lambda}:=\frac{\partial F_{\alpha}^{A}}{\partial p_{\Lambda}^{B}} .
$$

We will also use the summation convention that an index that appears both as a suband superscript is summed over. The functions in (1.6) appearing in the compatibility conditions (1.4) and (1.5) can now be written in the following way

$$
\Phi_{\alpha \beta}^{A}:=F_{\alpha \beta}^{A}+F_{\alpha B}^{A} F_{\beta}^{B}+F_{\alpha B}^{A \Gamma}\left(F_{\beta \Gamma}^{B}+F_{\beta C}^{B} p_{\Gamma}^{C}\right), \quad \Psi_{\alpha \beta C}^{A \Gamma \Lambda}:=F_{\alpha B}^{A \Gamma} F_{\beta C}^{B \Lambda}+F_{\alpha B}^{A \Lambda} F_{\beta C}^{B \Gamma} .
$$

\section{Solvability AND COMPATIBILITy CONDITIONS}

We keep the notation introduced in the previous section. We begin by proving the implication (i) $\Longrightarrow$ (iii) in Theorem 1.1.

Proof of (i) $\Longrightarrow$ (iii). If $u^{A}\left(x^{i}\right)$ is a solution to (2.1), in particular, of the equation

$$
u_{\alpha}^{A}\left(x^{i}\right)=F_{\alpha}^{A}\left(x^{i}, u^{B}\left(x^{i}\right), u_{\Gamma}^{B}\left(x^{i}\right)\right),
$$


then, by differentiating with respect to $x^{\Lambda}$, we obtain

$$
u_{\alpha \Lambda}^{A}=F_{\alpha \Lambda}^{A}+F_{\alpha B}^{A} u_{\Lambda}^{B}+F_{\alpha B}^{A \Gamma} u_{\Gamma \Lambda}^{B} .
$$

Similarly, differentiating (3.1) with respect to $x^{\beta}$, we obtain

$$
u_{\alpha \beta}^{A}=F_{\alpha \beta}^{A}+F_{\alpha B}^{A} u_{\beta}^{B}+F_{\alpha B}^{A \Gamma} u_{\Gamma \beta}^{B} .
$$

By substituting for $u_{\Gamma \beta}^{B}$ in (3.3) using (3.2) (and the symmetry in $\Gamma$ and $\beta$ ), we obtain

$$
u_{\alpha \beta}^{A}=F_{\alpha \beta}^{A}+F_{\alpha B}^{A} u_{\beta}^{B}+F_{\alpha B}^{A \Gamma}\left(F_{\beta \Gamma}^{B}+F_{\beta C}^{B} u_{\Gamma}^{C}+F_{\beta C}^{B \Omega} u_{\Omega \Gamma}^{C}\right) .
$$

In particular, since the left hand side is symmetric in $\alpha$ and $\beta$, it follows that the right hand side is also symmetric. Fix a point $\left(\left(x^{i}\right)_{0},\left(p^{A}\right)_{0},\left(p_{\Lambda}^{A}\right)_{0}\right) \in U$. By assumption, the Cauchy problem (2.1) has a unique solution for any Cauchy data $a^{A}\left(x^{\Lambda}\right)$ satisfying (2.2). We choose the data $a^{A}$ such that the second derivatives $a_{\Omega \Gamma}^{A}\left(x_{0}^{\Lambda}\right)$ vanish for all $A, \Omega$, and $\Gamma$. Evaluating (3.4) at $x^{i}=x_{0}^{i}$ and using the facts that

$$
u^{A}\left(x_{0}^{i}\right)=a^{A}\left(x_{0}^{\Lambda}\right)=\left(p^{A}\right)_{0}, \quad u_{\Gamma}^{A}\left(x_{0}^{i}\right)=a_{\Gamma}^{A}\left(x_{0}^{\Lambda}\right)=\left(p_{\Gamma}^{A}\right)_{0}, \quad u_{\Omega \Gamma}^{A}\left(x_{0}^{i}\right)=a_{\Omega \Gamma}^{A}\left(x_{0}^{\Lambda}\right)=0,
$$

we conclude that the quantity

$$
F_{\alpha \beta}^{A}+F_{\alpha B}^{A} p_{\beta}^{B}+F_{\alpha B}^{A \Gamma}\left(F_{\beta \Gamma}^{B}+F_{\beta C}^{B} p_{\Gamma}^{C}\right),
$$

evaluated at the point $\left(\left(x^{i}\right)_{0},\left(p^{A}\right)_{0},\left(p_{\Lambda}^{A}\right)_{0}\right)$, is symmetric in $\alpha$ and $\beta$. Since the point $\left(\left(x^{i}\right)_{0},\left(p^{A}\right)_{0},\left(p_{\Lambda}^{A}\right)_{0}\right)$ was arbitrary in $U$, we conclude that (1.4) holds identically in $U$.

To prove (1.5), we again fix a point $\left(\left(x^{i}\right)_{0},\left(p^{A}\right)_{0},\left(p_{\Lambda}^{A}\right)_{0}\right) \in U$ and choose the Cauchy data $a^{A}\left(x^{\Lambda}\right)$, satisfying (2.2), such that the second derivatives $a_{\Omega \Gamma}^{A}\left(x_{0}^{\Lambda}\right)$ are equal to arbitrary chosen real numbers $q_{\Omega \Gamma}^{A}$ with $q_{\Omega \Gamma}^{A}$ being symmetric in $\Omega$ and $\Gamma$. Evaluating (3.4) at $\left(x^{i}\right)_{0}$ and using the symmetry of (1.4) in $\alpha$ and $\beta$ as well as (3.5), we conclude that

$$
\left(F_{\alpha B}^{A \Gamma} F_{\beta C}^{B \Omega}-F_{\beta B}^{A \Gamma} F_{\alpha C}^{B \Omega}\right) q_{\Omega \Gamma}^{C}=0 .
$$

The symmetry condition given by (1.5) now follows, since the $q_{\Omega \Gamma}^{C}$ (symmetric in $\Omega$ and $\Gamma)$ and the point $\left(x_{0}^{i},\left(p^{A}\right)_{0},\left(p_{\Lambda}^{A}\right)_{0}\right) \in U$ are arbitrary. This completes the proof of $(\mathrm{i}) \Longrightarrow$ (iii).

Proof of (ii) $\Longleftrightarrow$ (iii). In order to prove the equivalence (ii) $\Longleftrightarrow$ (iii), we shall need the following proposition.

Proposition 3.1. Let $\left(\left(x^{i}\right)_{0},\left(p^{A}\right)_{0},\left(p_{\Lambda}^{A}\right)_{0}\right) \in U$ and $a^{A}\left(x^{\Lambda}\right)$ be real-analytic initial data defined in a neighborhood of $\left(x^{\Lambda}\right)_{0}$ satisfying (2.2). Then $u^{A}\left(x^{i}\right)$ is an approximate solution to (2.1) at $\left(x^{i}\right)_{0}$ if and only if the following equalities hold:

$$
\begin{aligned}
u^{A} & =a^{A}, \quad u_{\Gamma}^{A}=a_{\Gamma}^{A}, \quad u_{\Gamma \Omega}^{A}=a_{\Gamma \Omega}^{A}, \\
u_{\alpha}^{A} & =F_{\alpha}^{A} \\
u_{\alpha \Lambda}^{A} & =F_{\alpha \Lambda}^{A}+F_{\alpha B}^{A} a_{\Lambda}^{B}+F_{\alpha B}^{A \Gamma} a_{\Gamma \Lambda}^{B} \\
u_{\alpha \beta}^{A} & =F_{\alpha \beta}^{A}+F_{\alpha B}^{A} F_{\beta}^{B}+F_{\alpha B}^{A \Gamma}\left(F_{\beta \Gamma}^{B}+F_{\beta C}^{B} a_{\Gamma}^{C}+F_{\beta C}^{B \Omega} a_{\Omega \Lambda}^{C}\right),
\end{aligned}
$$


where all the $u^{A}$ (resp. $a^{A}$, resp. $F_{\alpha}^{A}$ ) and their derivatives appearing in the right hand sides of (3.8) are evaluated at the point $\left(x^{i}\right)_{0}$ (resp. $\left(x^{\Lambda}\right)_{0}$, resp. $\left.\left(\left(x^{i}\right)_{0},\left(p^{A}\right)_{0},\left(p_{\Lambda}^{A}\right)_{0}\right)\right)$.

Proof. Clearly, the first row of equations in (3.8) is equivalent to the initial condition in (1.3). Now, proceeding as in the proof of (i) $\Longrightarrow$ (iii) above, it is straightforward to verify that $u^{A}\left(x^{i}\right)$ satisfies the first row in (1.3) if and only if the remaining equations in (3.8) hold. This completes the proof of Proposition 3.1 .

The following proposition follows immediately from Proposition 3.1 by similar arguments to those used to prove (i) $\Longrightarrow$ (iii) above.

Proposition 3.2. Let $\left(\left(x^{i}\right)_{0},\left(p^{A}\right)_{0},\left(p_{\Lambda}^{A}\right)_{0}\right) \in U$. Then, the following are equivalent:

(ii') The Cauchy problem (2.1) is approximately solvable for every initial data at $\left(\left(x^{i}\right)_{0},\left(p^{A}\right)_{0},\left(p_{\Lambda}^{A}\right)_{0}\right)$.

(iii') The functions $F_{\alpha}^{A}$ satisfy the compatibility conditions (1.4) and (1.5) at the point $\left(\left(x^{i}\right)_{0},\left(p^{A}\right)_{0},\left(p_{\Lambda}^{A}\right)_{0}\right)$.

Clearly, the equivalence (ii) $\Longleftrightarrow$ (iii) in Theorem 1.1 is a direct consequence of Proposition 3.2 by letting the point $\left(\left(x^{i}\right)_{0},\left(p^{A}\right)_{0},\left(p_{\Lambda}^{A}\right)_{0}\right)$ vary in $U$.

\section{Approximate solvability with Respect to A slope}

We begin this section by considering a somewhat more general system of first order partial differential equations, using still the conventions introduced in previous sections:

$$
G_{\alpha}^{A}\left(x^{i}, u^{B}, u_{i}^{B}\right)=0, \quad \alpha=k+1, \ldots, n, A=1, \ldots, m,
$$

where $G_{\alpha}^{A}$ are real-analytic functions defined in an open subset $V$ of $\mathbb{R}^{n} \times \mathbb{R}^{m} \times \mathbb{R}^{n m}$ and $\left(\left(x^{i}\right)_{0},\left(p^{A}\right)_{0},\left(p_{i}^{A}\right)_{0}\right) \in V$ is such that $G_{\alpha}^{A}\left(\left(x^{i}\right)_{0},\left(p^{A}\right)_{0},\left(p_{i}^{A}\right)_{0}\right)=0$. Let $M$ a $k$-dimensional (real-analytic) submanifold in $\mathbb{R}^{n}$ through $x_{0}$ whose tangent $k$-plane $P$ at $x_{0}$ is given by

$$
P=T_{x_{0}} M=\left\{x^{i}:\left(x^{j}-x_{0}^{j}\right) \xi_{j}^{\beta}=0, \beta=k+1, \ldots, n\right\},
$$

where $\xi^{\beta}:=\left(\xi_{i}^{\beta}\right)_{i=1, \ldots, n}$ are linearly independent vectors in $\mathbb{R}^{n}$. We shall say that $M$ is non-characteristic at $\left(\left(x^{i}\right)_{0},\left(p^{A}\right)_{0},\left(p_{i}^{A}\right)_{0}\right)$ for the system (4.1) if the $m(n-k) \times m(n-k)$ matrix $W:=\left(W_{B, \alpha}^{A, \beta}\right)$, where $A, B$ run over $\{1, \ldots m\}$ and $\alpha, \beta$ over $\{k+1, \ldots, n\}$, is invertible with

$$
W_{B, \alpha}^{A, \beta}:=G_{\alpha B}^{A j}\left(\left(x^{i}\right)_{0},\left(p^{A}\right)_{0},\left(p_{i}^{A}\right)_{0}\right) \xi_{j}^{\beta},
$$

where we continue using our convention that adding extra indices corresponds to derivatives, i.e. $G_{\alpha B}^{A j}$ is the derivative of $G_{\alpha}^{A}$ in $p_{j}^{B}$.

Proposition 4.1. Suppose that a $k$-dimensional real-analytic submanifold $M$ of $\mathbb{R}^{n}$ through $x_{0}$ is non-characteristic at $\left(\left(x^{i}\right)_{0},\left(p^{A}\right)_{0},\left(p_{i}^{A}\right)_{0}\right)$ for the system (4.1). Then after a 
real-analytic change of coordinates in $\mathbb{R}^{n}$ near $x_{0}$ preserving $x_{0}, M$ can be written near $x_{0}$ as $\left\{x: x^{\alpha}=x_{0}^{\alpha}, \alpha=k+1, \ldots, n\right\}$ and the system (4.1) near $\left(\left(x^{i}\right)_{0},\left(p^{A}\right)_{0},\left(p_{i}^{A}\right)_{0}\right)$ as

$$
u_{\alpha}^{A}=F_{\alpha}^{A}\left(x^{i}, u^{B}, u_{\Lambda}^{B}\right), \quad A=1 \ldots, m, \alpha=k+1, \ldots n,
$$

where $F_{\alpha}^{A}\left(x^{i}, p^{B}, p_{\Lambda}^{B}\right)$ is real-analytic near the point $\left(\left(x^{i}\right)_{0},\left(p^{A}\right)_{0},\left(p_{\Lambda}^{A}\right)_{0}\right)$ with $\left(p_{\alpha}^{A}\right)_{0}=$ $F_{\alpha}^{A}\left(\left(x^{i}\right)_{0},\left(p^{B}\right)_{0},\left(p_{\Lambda}^{B}\right)_{0}\right)$.

Proof. Let $\left(\xi^{\beta}\right)$ be as in (4.2) and $\left(\xi^{\Lambda}\right)$ be further vectors in $\mathbb{R}^{n}$ such that the collection $\left(\xi^{i}\right)$ forms a basis for $\mathbb{R}^{n}$ and let $\xi$ denote the invertible $n \times n$ matrix whose $i$-th row equals $\xi^{i}$. Then there exists a real-analytic change of coordinates near $x_{0}$ of the form

$$
\tilde{x}^{i}:=x_{0}^{i}+\xi_{j}^{i}\left(x^{j}-x_{0}^{j}\right)+O\left(\sum_{j}\left|x^{j}-x_{0}^{j}\right|^{2}\right),
$$

such that $M$ near $x_{0}$ is given by $\tilde{x}^{\alpha}=\tilde{x}_{0}^{\alpha}$, where $\tilde{x}_{0}^{i}=x_{0}^{i}$. Let $\eta$ denote the inverse of the matrix $\xi$. Then, an application of the chain rule shows that in the new coordinates, the system (4.1) takes the form $\tilde{G}_{\alpha}^{A}\left(\tilde{x}^{i}, \tilde{u}^{B}, \tilde{u}_{i}^{B}\right)=0$, where

$$
\tilde{G}_{\alpha}^{A}\left(\tilde{x}^{i}, \tilde{p}^{B}, \tilde{p}_{i}^{B}\right)=G_{\alpha}^{A}\left(x_{0}^{i}+\eta_{j}^{i}\left(\tilde{x}^{j}-x_{0}^{j}\right), \tilde{p}^{B}, \xi_{i}^{j} \tilde{p}_{j}^{B}\right)+O\left(\sum_{j}\left|x^{j}-x_{0}^{j}\right|^{2}\right) .
$$

Note that $\tilde{G}_{\alpha B}^{A \beta}\left(\left(\tilde{x}^{i}\right)_{0},\left(\tilde{p}^{A}\right)_{0},\left(\tilde{p}_{i}^{A}\right)_{0}\right)=W_{B, \alpha}^{A, \beta}$, the latter given by (4.3). Thus, the conclusion of Proposition 4.1 follows immediately from the implicit function theorem.

To prove (ii) $\Longrightarrow(\mathrm{i})$ in Theorem 1.1, we shall need some further preliminary material. Let $F_{\alpha}^{A}$ be as in previous sections, $\left(\left(x^{i}\right)_{0},\left(p^{A}\right)_{0},\left(p_{\Lambda}^{A}\right)_{0}\right)$ be a point in $U,\left(c_{\Lambda}^{\alpha}\right)$ be a real $(n-k) \times k$ matrix, and $P \subset \mathbb{R}^{n}$ the $k$-plane through the point $x_{0}$ given by

$$
P:=\left\{x: x^{\alpha}=x_{0}^{\alpha}+c_{\Lambda}^{\alpha}\left(x^{\Lambda}-x_{0}^{\Lambda}\right), \alpha=k+1, \ldots, n\right\} .
$$

Note that $P$, given by (4.7), is non-characteristic at $\left(\left(x^{i}\right)_{0},\left(p^{A}\right)_{0},\left(p_{i}^{A}\right)_{0}\right)$ with $\left(p_{\alpha}^{A}\right)_{0}=$ $F_{\alpha}^{A}\left(\left(x^{i}\right)_{0},\left(p^{A}\right)_{0},\left(p_{\Lambda}^{A}\right)_{0}\right)$ for the system (4.4) if the $m(n-k) \times m(n-k)$ matrix $V:=\left(V_{B, \alpha}^{A, \beta}\right)$ (where as before $A, B$ run over $\{1, \ldots m\}$ and $\alpha, \beta$ over $\{k+1, \ldots, n\}$ ) is invertible with

$$
V_{B, \alpha}^{A, \beta}:=\delta_{B}^{A} \delta_{\alpha}^{\beta}-F_{\alpha B}^{A \Lambda}\left(\left(x^{i}\right)_{0},\left(p^{A}\right)_{0},\left(p_{\Lambda}^{A}\right)_{0}\right) c_{\Lambda}^{\beta},
$$

where $\delta_{j}^{i}$ is the standard Kronecker delta symbol. In this case, we shall say that the slope $\left(c_{\Lambda}^{\alpha}\right)$ is non-characteristic at $\left(\left(x^{i}\right)_{0},\left(p^{A}\right)_{0},\left(p_{\Lambda}^{A}\right)_{0}\right)$ for the system (4.4).

For non-characteristic slopes $\left(c_{\Lambda}^{\alpha}\right)$, we shall consider the Cauchy problem for

$$
u_{\alpha}^{A}=F_{\alpha}^{A}\left(x^{i}, u^{B}, u_{\Lambda}^{B}\right)
$$

with data on the tilted $k$-plane (4.7). We shall say that this Cauchy problem with respect to the (non-characteristic) slope $\left(c_{\Lambda}^{\alpha}\right)$ is approximately solvable for every initial data at $\left(\left(x^{i}\right)_{0},\left(p^{A}\right)_{0},\left(p_{\Lambda}^{A}\right)_{0}\right)$ if, for every real-analytic $a^{A}\left(x^{\Lambda}\right)$ defined in a neighborhood of $\left(x^{\Lambda}\right)_{0}$ and satisfying

$$
a^{A}\left(x_{0}^{\Gamma}\right)=\left(p^{A}\right)_{0}, \quad a_{\Lambda}^{A}\left(x_{0}^{\Gamma}\right)=\left(p_{\Lambda}^{A}\right)_{0}+F_{\alpha}^{A}\left(\left(x^{i}\right)_{0},\left(p^{A}\right)_{0},\left(p_{\Omega}^{A}\right)_{0}\right) c_{\Lambda}^{\alpha},
$$


there is an approximate solution $u^{A}\left(x^{i}\right)$ at $x_{0}$ to the Cauchy problem

$$
\left\{\begin{array}{l}
u_{\alpha}^{A}=F_{\alpha}^{A}\left(x^{i}, u^{B}, u_{\Gamma}^{B}\right) \\
u^{A}\left(x^{\Lambda}, x_{0}^{\beta}+c_{\Gamma}^{\beta}\left(x^{\Gamma}-x_{0}^{\Gamma}\right)\right)=a^{A}\left(x^{\Lambda}\right) .
\end{array}\right.
$$

(That is, the first equation in (4.11) holds up to order 1 at $\left(x^{i}\right)_{0}$ and the second up to order 2 at $\left(x^{\Lambda}\right)_{0}$.) Note that (4.10) is a modification of (2.2) corresponding to the tilted plane (4.7). This choice also has the effect that the definition of approximate solvability with respect to a slope is invariant under linear changes of coordinates. In the terminology of the previous section, the approximate solvability of the Cauchy problem corresponds to that with respect to the slope $\left(c_{\Lambda}^{\alpha}\right)$ with $c_{\Lambda}^{\alpha}=0$.

The following statement relates approximate solvability with respect to different slopes and is of independent interest.

Proposition 4.2. The Cauchy problem for (4.9) with respect to a fixed non-characteristic slope is approximately solvable for every initial data at $\left(\left(x^{i}\right)_{0},\left(p^{A}\right)_{0},\left(p_{\Lambda}^{A}\right)_{0}\right)$ if and if it is approximately solvable for every initial data at $\left(\left(x^{i}\right)_{0},\left(p^{A}\right)_{0},\left(p_{\Lambda}^{A}\right)_{0}\right)$ with respect to every non-characteristic slope.

Proof. We observe that all results proved so far also hold in the complex-analytic setting, i.e. by letting the variables $\left(x^{i}, p^{B}, p_{\Lambda}^{B}\right)$ be complex and the functions $F_{\alpha}^{A}, u^{A}$, and the initial data $a^{A}$ be holomorphic with respect to their arguments. In this proof, we shall first consider this complex setting. Thus, all variables will be understood to be complex and all functions holomorphic. Let $a^{A}\left(x^{\Lambda}\right)$ be any data satisfying (4.10). As in Proposition 3.1 and its proof, a function $u^{A}\left(x^{i}\right)$ is an approximate solution to (4.11) at $\left(\left(x^{i}\right)_{0},\left(p^{A}\right)_{0},\left(p_{\Lambda}^{A}\right)_{0}\right)$ (for $a^{A}$ satisfying (4.10) ) if and only if the following equalities hold:

$$
\begin{aligned}
u^{A} & =a^{A}, \quad u_{\Gamma}^{A}+F_{\alpha}^{A} c_{\Gamma}^{\alpha}=a_{\Gamma}^{A}, \quad u_{\Lambda \Gamma}^{A}+u_{\Lambda \beta}^{A} c_{\Gamma}^{\beta}+u_{\alpha \Gamma}^{A} c_{\Lambda}^{\alpha}+u_{\alpha \beta}^{A} c_{\Lambda}^{\alpha} c_{\Gamma}^{\beta}=a_{\Lambda \Gamma}^{A} \\
u_{\alpha}^{A} & =F_{\alpha}^{A} \\
u_{\alpha \Lambda}^{A} & =F_{\alpha \Lambda}^{A}+F_{\alpha B}^{A}\left(p_{\Lambda}^{B}\right)_{0}+F_{\alpha B}^{A \Gamma} u_{\Gamma \Lambda}^{B} \\
u_{\alpha \beta}^{A} & =F_{\alpha \beta}^{A}+F_{\alpha B}^{A} F_{\beta}^{B}+F_{\alpha B}^{A \Gamma}\left(F_{\beta \Gamma}^{B}+F_{\beta C}^{B}\left(p_{\Gamma}^{C}\right)_{0}+F_{\beta C}^{B \Omega} u_{\Omega \Lambda}^{C}\right),
\end{aligned}
$$

where all the $u^{A}$ (resp. $a^{A}$, resp. $F_{\alpha}^{A}$ ) and their derivatives appearing in the right hand sides are evaluated at the point $\left(x^{i}\right)_{0}$ (resp. $\left(x^{\Lambda}\right)_{0}$, resp. $\left.\left(\left(x^{i}\right)_{0},\left(p^{A}\right)_{0},\left(p_{\Lambda}^{A}\right)_{0}\right)\right)$. We observe that, for small values of the slope $c_{\Lambda}^{\alpha}$, there is always a unique solution to (4.12) consisting of the value of $u^{A}$ and its derivatives up to order 2 at $x_{0}$. To see this, notice that the system is trivial to solve when $c_{\Lambda}^{\alpha}=0$. As in the proof of (i) $\Longrightarrow$ (iii) above (cf. Proposition 3.2), we see that for $\left(c_{\Lambda}^{\alpha}\right)$ sufficiently small, (4.12) implies (1.4) and (1.5). Indeed $u_{\alpha \beta}=u_{\beta \alpha}$ implies the symmetry in $\alpha$ and $\beta$ of the right-hand side of the last identity in (4.12). Furthermore, for $c_{\Lambda}^{\alpha}$ small, as the $a_{\Lambda \Gamma}^{A}$ vary over all possible choices, so do the $u_{\Lambda \Gamma}^{A}$ satisfying (4.12). Hence we obtain the compatibility conditions (1.4) and (1.5) at $\left(\left(x^{i}\right)_{0},\left(p^{A}\right)_{0},\left(p_{\Lambda}^{A}\right)_{0}\right)$. Vice versa, if (1.4) and (1.5) hold at $\left(\left(x^{i}\right)_{0},\left(p^{A}\right)_{0},\left(p_{\Lambda}^{A}\right)_{0}\right)$, the 
right-hand side of the last identity in (4.12) is always symmetric in $\alpha$ and $\beta$. Hence the system (4.12) is always solvable in $u_{\Lambda \Gamma}^{A}, u_{\alpha \Lambda}^{A}, u_{\alpha \beta}^{A}$ for $c_{\Lambda}^{\alpha}$ small.

In particular, we conclude that approximate solvability for every initial data at $\left(\left(x^{i}\right)_{0},\left(p^{A}\right)_{0},\left(p_{\Lambda}^{A}\right)_{0}\right)$ with respect to one sufficiently small slope $\left(c_{\Lambda}^{\alpha}\right)$ is equivalent to approximate solvability for every initial data at $\left(\left(x^{i}\right)_{0},\left(p^{A}\right)_{0},\left(p_{\Lambda}^{A}\right)_{0}\right)$ with respect to every sufficiently small slope $\left(c_{\Lambda}^{\alpha}\right)$. We claim that this fact implies that the set $\mathcal{S}$ of all non-characteristic complex slopes $\left(c_{\Lambda}^{\alpha}\right)$ with respect to which approximate solvability (for every initial data) holds at $\left(\left(x^{i}\right)_{0},\left(p^{A}\right)_{0},\left(p_{\Lambda}^{A}\right)_{0}\right)$ is open and closed in the space of all non-characteristic slopes. Indeed, given any non-characteristic $\left(c_{\Lambda}^{\alpha}\right)$, then, after a linear change of coordinates of the form $\tilde{x}^{\Lambda}=x^{\Lambda}, \tilde{x}^{\alpha}=x^{\alpha}-c_{\Lambda}^{\alpha}\left(x^{\Lambda}-x_{0}^{\Lambda}\right)$, the initial $k$-plane (4.7) becomes $\left\{\tilde{x}^{\alpha}=x_{0}^{\alpha}\right\}$ and the system of differential equation (4.9) can be written as $\tilde{u}_{\alpha}^{A}=\tilde{F}_{\alpha}^{A}\left(\tilde{x}^{i}, \tilde{u}^{B}, \tilde{u}_{\Lambda}^{B}\right)$ by Proposition 4.1. Since approximate solvability with respect to slopes is invariant under affine changes of coordinates, the observation above shows that $\mathcal{S}$ is both open and closed in the space of all non-characteristic slopes. Now, observe that the set of all non-characteristic slopes is Zariski-open in the space of all complex $(n-k) \times k$ matrices. In particular, this set is connected, which by the above proves that the set $\mathcal{S}$ is either empty or equals the whole set of non-characteristic complex slopes. By specializing to real non-characteristic slopes, the proposition follows.

\section{EXTERIOR DIFFERENTIAL SYSTEMS AND INTEGRAL MANIFOLDS}

In this section, we shall reformulate solvability of the Cauchy problem (2.1) in terms of the existence and uniqueness of integral manifolds for an exterior differential system. The reader is referred to the text $\left[\mathrm{BCG}^{3} 91\right]$ for further details of the theory of exterior differential systems and their integral manifolds. We here briefly recall the needed terminology in the real-analytic case. On a real-analytic manifold $X$, consider the graded ring of all real-analytic exterior differential forms (of any degree) on $X$ with respect to the exterior product. That is, an element of the ring is a (formal) finite sum of exterior forms of different degrees. A real-analytic exterior differential system is any differential ideal $\mathcal{I}$ in this ring, i.e. any algebraic ideal that is closed under exterior differentiation. A real submanifold $S \subset X$ is an integral submanifold of $\mathcal{I}$ if every element from $\mathcal{I}$ vanishes when restricted to $S$. Here a formal sum of exterior differential forms of different degrees is said to vanish on $S$ if each homogeneous component of the formal sum does.

Now consider a solution $u^{A}\left(x^{i}\right)$ to the system of partial differential equations

$$
u_{\alpha}^{A}\left(x^{i}\right)=F_{\alpha}^{A}\left(x^{i}, u^{B}\left(x^{i}\right), u_{\Gamma}^{B}\left(x^{i}\right)\right),
$$

with $F_{\alpha}^{A}$ being real-analytic in an open subset $U \subset \mathbb{R}^{n} \times \mathbb{R}^{m} \times \mathbb{R}^{k m}$. Then its jet-graph

$$
S_{u^{A}}:=\left\{\left(x^{i}, p^{B}, p_{j}^{B}\right): p^{B}=u^{B}\left(x^{i}\right), p_{j}^{B}=u_{j}^{B}\left(x^{i}\right)\right\} \subset \mathbb{R}^{n} \times \mathbb{R}^{m} \times \mathbb{R}^{n m},
$$

is an integral submanifold for the exterior differential system $\mathcal{I}$ generated by the forms

$$
p_{\alpha}^{A}-F_{\alpha}^{A}\left(x^{i}, p^{B}, p_{\Gamma}^{B}\right), \quad d p^{B}-p_{i}^{B} d x^{i},
$$


defined in

$$
\tilde{U}:=U \times \mathbb{R}^{(n-k) m} \subset \mathbb{R}^{n} \times \mathbb{R}^{m} \times \mathbb{R}^{n m} .
$$

Conversely, if $S \subset \tilde{U}$ is an integral submanifold of $\mathcal{I}$ such that $d x^{1} \wedge \ldots \wedge d x^{n} \neq 0$ everywhere on $S$, then $S$ is automatically $n$-dimensional and is locally a jet-graph $S_{u^{A}}$ of a solution $u^{A}\left(x^{i}\right)$ to (5.1).

The differential ideal $\mathcal{I}$ is generated algebraically by the differential forms

$$
\left\{\begin{aligned}
f_{\alpha}^{A} & :=p_{\alpha}^{A}-F_{\alpha}^{A}\left(x^{i}, p^{B}, p_{\Gamma}^{B}\right), \\
\omega_{\alpha}^{A} & :=d f_{\alpha}^{A}, \\
\eta^{A} & :=d p^{A}-p_{i}^{A} d x^{i}, \\
\Omega^{A} & :=-d \eta^{A}=d p_{i}^{A} \wedge d x^{i} .
\end{aligned}\right.
$$

For convenience of notation, we shall write $z=\left(x^{i}, p^{B}, p_{i}^{B}\right)$ and hence $z_{0}$ will denote the point $\left(\left(x^{i}\right)_{0}, p_{0}^{B},\left(p_{i}^{B}\right)_{0}\right)$.

Recall that if $E$ is a linear $l$-dimensional subspace of $T_{z} \mathbb{R}^{n+m+n m}$, then $E$ is called an integral element of $\mathcal{I}$ if for all $p$-forms $\phi \in \mathcal{I}$, we have $\phi\left(v_{1}, \ldots, v_{p}\right)=0$ for all $v_{1}, \ldots, v_{p} \in$ $E$. If $E$ is an integral element, then its polar space (with respect to the differential ideal $\mathcal{I})$, denoted by $H(E)$, is given by

$$
H(E):=\left\{v \in T_{z} \mathbb{R}^{n+m+n m}: \phi\left(v, e_{1}, \ldots, e_{l}\right)=0, \forall(l+1) \text {-forms } \phi \text { in } \mathcal{I}\right\},
$$

where $e_{1}, \ldots, e_{l}$ is a basis for $E$.

Remark 5.1. It follows directly from the definition that if $E$ is an integral element, it is always contained in $H\left(E^{\prime}\right)$ for any $E^{\prime} \subset E$.

We shall use the following elementary lemma, whose proof is left to the reader.

Lemma 5.2. Let $E \subset T_{z} \mathbb{R}^{n+m+n m}$ be an integral element of $\mathcal{I}$. Then, a vector $v$ is in $H(E)$ if and only if

$$
\omega_{\alpha}^{A}(v)=0, \eta^{A}(v)=0, \Omega^{A}(v, e)=0, \quad \forall e \in E .
$$

We shall also need the following proposition.

Proposition 5.3. Let $E \subset T_{z} \mathbb{R}^{n+m+n m}$ be an integral element. Assume that there are vectors $v_{1}, \ldots, v_{k} \in E$ such that

$$
\left(d x^{1} \wedge \ldots \wedge d x^{k}\right)\left(v_{1}, \ldots, v_{k}\right) \neq 0
$$

and such that the push forwards of these vectors by the projection $\pi: \mathbb{R}^{n+m+n m} \rightarrow \mathbb{R}^{n}$, given by $\pi\left(x^{i}, p^{A}, p_{j}^{A}\right)=\left(x^{i}\right)$, span a non-characteristic $k$-plane of (5.1) at $z$. Then $\operatorname{dim} H(E) \leq n$. 
Proof. The assumption (5.8) is equivalent to the fact that there are $k$ vectors in the span of $v_{1}, \ldots, v_{k}$ of the form

$$
e_{\Lambda}=\frac{\partial}{\partial x^{\Lambda}}+e_{\Lambda}^{\alpha} \frac{\partial}{\partial x^{\alpha}}+e_{\Lambda}^{A} \frac{\partial}{\partial p^{A}}+e_{\Lambda j}^{A} \frac{\partial}{\partial p_{j}^{A}} .
$$

The assumption that the span of $v_{1}, \ldots, v_{k}$ projects onto a noncharacteristic $k$-plane means that the slope $\left(c_{\Lambda}^{\alpha}\right):=\left(e_{\Lambda}^{\alpha}\right)$ is noncharacteristic at $z$, i.e. the matrix (4.8) is invertible with $\left(c_{\Lambda}^{\alpha}\right):=\left(e_{\Lambda}^{\alpha}\right)$. Let $v$ be given by

$$
v=d^{j} \frac{\partial}{\partial x^{j}}+d^{A} \frac{\partial}{\partial p^{A}}+d_{j}^{A} \frac{\partial}{\partial p_{j}^{A}} .
$$

By Lemma 5.2, if $v$ belongs to $H(E)$, then (5.7) holds, in particular, for all $e=e_{\Lambda}$. A straightforward calculation shows that the equations (5.7) with $e=e_{\Lambda}$ are given by

$$
\begin{aligned}
\omega_{\alpha}^{A}(v) & =d_{\alpha}^{A}-d^{j} F_{\alpha j}^{A}-d^{B} F_{\alpha B}^{A}-d_{\Lambda}^{B} F_{\alpha B}^{A \Lambda}=0 \\
\eta^{A}(v) & =d^{A}-d^{i} p_{i}^{A}=0 \\
\Omega^{A}\left(v, e_{\Lambda}\right) & =d_{\Lambda}^{A}+d_{\alpha}^{A} e_{\Lambda}^{\alpha}-e_{\Lambda i}^{A} d^{i}=0 .
\end{aligned}
$$

If we now solve for $d^{A}$ in the second line of (5.11), for $d_{\Lambda}^{A}$ in the third line, and substitute the result in the first line, we obtain equations for $d_{\alpha}^{A}$ of the form

$$
\left(\delta_{B}^{A} \delta_{\alpha}^{\beta}-F_{\alpha B}^{A \Lambda} e_{\Lambda}^{\beta}\right) d_{\beta}^{B}=R_{\alpha}^{A},
$$

where the $R_{\alpha}^{A}$ depend only on the $d^{i}$. Since the matrix (4.8) is invertible with $\left(c_{\Lambda}^{\alpha}\right):=\left(e_{\Lambda}^{\alpha}\right)$, we conclude that we may solve (5.12) for $d_{\alpha}^{A}$ in terms of the $d^{i}$. By substituting back into the previously solved equations, we conclude that the coefficients $d^{A}, d_{i}^{A}$ are all determined by the coefficients $d^{i}$. This proves that $\operatorname{dim} H(E) \leq n$.

For $z_{0}:=\left(\left(x^{i}\right)_{0}, p_{0}^{B},\left(p_{i}^{B}\right)_{0}\right) \in \tilde{U}$ (given by (5.4)), let $u^{A}(x)$ be a real-analytic function satisfying $u^{A}\left(x_{0}\right)=\left(p^{A}\right)_{0}$ and $u_{j}^{A}\left(x_{0}\right)=\left(p_{j}^{A}\right)_{0}$. Denote again by $S_{u^{A}}$ its jet-graph, given by (5.2). Observe that the exterior forms $\eta^{A}$ and $\Omega^{A}=d \eta^{A}$ in (5.5) vanish identically on the (any) jet-graph $S_{u^{A}}$. Also, note that $u^{A}$ is an approximate solution to the system (5.1) at $x_{0}$ if and only if the restriction of the functions $f_{\alpha}^{A}$ in (5.5) to $S_{u^{A}}$ vanishes up to second order at $z_{0}$ (see (1.3) ). Consequently, $u^{A}$ is an approximate solution to the system (5.1) at $x_{0}$ if and only if the tangent space $T_{z_{0}} S_{u^{A}}$ is an integral element of the differential ideal $\mathcal{I}$ generated by (5.5). Summarizing and using Remark 5.1, we obtain the following proposition.

Proposition 5.4. Let $z_{0}:=\left(\left(x^{i}\right)_{0}, p_{0}^{B},\left(p_{i}^{B}\right)_{0}\right) \in \tilde{U}$ and assume that $S_{u^{A}}$ is a jet-graph through $z_{0}$ of an approximate solution $u^{A}$ to (5.1) at $x_{0}$. Then $T_{z_{0}} S_{u^{A}}$ is an integral element of $\mathcal{I}$. If $E \subset T_{z_{0}} S_{u^{A}}$, then $T_{z_{0}} S_{u^{A}} \subset H(E)$.

Combining Propositions 5.3 and 5.4, we obtain the following: 
Corollary 5.5. Let $z_{0}:=\left(\left(x^{i}\right)_{0}, p_{0}^{B},\left(p_{i}^{B}\right)_{0}\right) \in \tilde{U}$ and assume that $S_{u^{A}}$ is a jet-graph through $z_{0}$ of an approximate solution $u^{A}$ to (5.1) at $x_{0}$. If $E \subset T_{z_{0}} S_{u^{A}}$ and the assumption of Proposition 5.3 holds, then $T_{z_{0}} S_{u^{A}}=H(E)$.

Before entering the proof of (ii) $\Longrightarrow$ (i) in Theorem 1.1, we shall need one more result.

Proposition 5.6. Assume that property (ii) of Theorem 1.1 holds. Let $z_{0}:=$ $\left(\left(x^{i}\right)_{0}, p_{0}^{B},\left(p_{i}^{B}\right)_{0}\right) \in \tilde{U}$ and $E \subset T_{z_{0}} \mathbb{R}^{n+m+n m}$ be an integral element of $\mathcal{I}$. Assume that there are vectors $v_{1}, \ldots, v_{k} \in E$ such that (5.8) holds and such that the push forwards of these vectors by the projection $\pi: \mathbb{R}^{n+m+n m} \rightarrow \mathbb{R}^{n}$, given by $\pi\left(x^{i}, p^{A}, p_{j}^{A}\right)=\left(x^{i}\right)$, span a non-characteristic $k$-plane of (5.1) at $z_{0}$. Then there exists an approximate solution $u^{A}\left(x^{i}\right)$ to (5.1) at $x_{0}$ such that its jet graph $S_{u^{A}}$ contains $z_{0}$ and $E \subset T_{z_{0}} S_{u^{A}}$.

Proof. After replacing the $k$ vectors $v_{1}, \ldots, v_{k}$ by suitable linear combinations, we may assume that there are vectors $e_{1}, \ldots, e_{k}$ of the form (5.9) whose push forward via $\pi$ span a non-characteristic $k$-plane in $\mathbb{R}^{n}$. The latter condition is equivalent to the slope $\left(c_{\Lambda}^{\alpha}\right)$, given by $c_{\Lambda}^{\alpha}=e_{\Lambda}^{\alpha}$, being non-characteristic at $z_{0}$. As in the proof of Proposition 4.2, we make the linear change of coordinates $\tilde{x}^{\Lambda}=x^{\Lambda}, \tilde{x}^{\alpha}=x^{\alpha}-c_{\Lambda}^{\alpha}\left(x^{\Lambda}-x_{0}^{\Lambda}\right)$ and observe that, in the new coordinates, the system of differential equations (5.1) can be written as $\tilde{u}_{\alpha}^{A}=\tilde{F}_{\alpha}^{A}\left(\tilde{x}^{i}, \tilde{u}^{B}, \tilde{u}_{\Lambda}^{B}\right)$ by Proposition 4.1 and the Cauchy problem for this equation is still approximately solvable for every initial data at $\left(\left(x^{i}\right)_{0},\left(p^{B}\right)_{0},\left(p_{\Lambda}^{B}\right)_{0}\right)$ in view of Proposition 4.2. Hence, without loss of generality, we may assume that the $k$ vectors $e_{\Lambda}$ are of the form

$$
e_{\Lambda}=\frac{\partial}{\partial x^{\Lambda}}+e_{\Lambda}^{A} \frac{\partial}{\partial p^{A}}+e_{\Lambda j}^{A} \frac{\partial}{\partial p_{j}^{A}} .
$$

The fact that $E$ is an integral element implies, using the notation (5.5), that we have the relations

$$
\begin{aligned}
\eta^{A}\left(e_{\Lambda}\right) & =e_{\Lambda}^{A}-\left(p_{\Lambda}^{A}\right)_{0}=0 \\
\Omega^{A}\left(e_{\Lambda}, e_{\Gamma}\right) & =e_{\Lambda \Gamma}^{A}-e_{\Gamma \Lambda}^{A}=0 \\
\omega_{\alpha}^{A}\left(e_{\Lambda}\right) & =e_{\Lambda \alpha}^{A}-F_{\alpha \Lambda}^{A}-F_{\alpha B}^{A} e_{\Lambda}^{B}-F_{\alpha B}^{A \Gamma} e_{\Lambda \Gamma}^{B}=0 .
\end{aligned}
$$

We now consider the Cauchy problem (2.1) with initial data $a^{A}$ satisfying (2.2) and $a_{\Lambda \Gamma}^{A}\left(\left(x^{\Lambda}\right)_{0}\right)=e_{\Lambda \Gamma}^{A}$. By assumption, this Cauchy problem has an approximate solution $u^{A}\left(x^{i}\right)$. Hence the identities (3.8) hold (when evaluated at suitable points as outlined below (3.8)). Let $S_{0}$ and $S_{u^{A}}$ denote the corresponding jet graphs, i.e. the two manifolds parametrized near $z_{0}$, respectively, by the two maps $\Phi: \mathbb{R}^{k} \rightarrow \mathbb{R}^{n+m+n m}$ and $\Psi: \mathbb{R}^{n} \rightarrow$ $\mathbb{R}^{n+m+n m}$, given by

$$
\begin{gathered}
\Phi\left(x^{\Lambda}\right):=\left(\left(x^{\Lambda}, x_{0}^{\alpha}\right), a^{A}\left(x^{\Lambda}\right),\left(a_{\Gamma}^{A}\left(x^{\Lambda}\right), F_{\alpha}^{A}\left(\left(x^{\Lambda}, x_{0}^{\alpha}\right), a^{B}\left(x^{\Lambda}\right), a_{\Gamma}^{B}\left(x^{\Lambda}\right)\right)\right)\right) \\
\Psi\left(x^{i}\right):=\left(x^{i}, u^{A}\left(x^{i}\right), u_{j}^{A}\left(x^{i}\right)\right) .
\end{gathered}
$$


In the following we consider standard tangent vectors $\partial / \partial x^{i} \in T_{x_{0}} \mathbb{R}^{n}$. Making use of the equations (5.14), it is straightforward to check that we have

$$
\Phi_{*}\left(\partial / \partial x^{\Lambda}\right)=\Psi_{*}\left(\partial / \partial x^{\Lambda}\right)=e_{\Lambda}, \quad \Lambda=1, \ldots, k,
$$

with $e_{\Lambda}$ being given by (5.13). Furthermore, we have

$$
e_{\alpha}:=\Psi_{*}\left(\partial / \partial x^{\alpha}\right)=\frac{\partial}{\partial x^{\alpha}}+u_{\alpha}^{A} \frac{\partial}{\partial p^{A}}+u_{\alpha \Lambda}^{A} \frac{\partial}{\partial p_{\Lambda}^{A}}+u_{\alpha \beta}^{A} \frac{\partial}{\partial p_{\beta}^{A}},
$$

where the coefficients $u_{\alpha}^{A}, u_{\alpha j}^{A}$ are evaluated at $x_{0}$ and satisfy the equations in (3.8). By the construction, the $n$ vectors $e_{1}, \ldots, e_{n}$ from (5.13) and (5.17) form a basis for $T_{z_{0}} S_{u^{A}}$. In order to show that $E \subset T_{z_{0}} S_{u^{A}}$, we let $w$ be a vector in $E$, which is not contained in the span of $e_{1}, \ldots, e_{k}$. Without loss of generality, we may assume that $w$ is of the form

$$
w=w^{\alpha} \frac{\partial}{\partial x^{\alpha}}+w^{A} \frac{\partial}{\partial p^{A}}+w_{\Lambda}^{A} \frac{\partial}{\partial p_{\Lambda}^{A}}+w_{\alpha}^{A} \frac{\partial}{\partial p_{\alpha}^{A}},
$$

for some real coefficients $w^{\alpha}, w^{A}, w_{j}^{A}$. The fact that $E$ is an integral element implies, in view of (5.5), that

$$
\begin{aligned}
\eta^{A}(w) & =w^{A}-\left(p_{\beta}^{A}\right)_{0} w^{\beta}=0, \\
\Omega^{A}\left(w, e_{\Lambda}\right) & =w_{\Lambda}^{A}-e_{\Lambda \beta}^{A} w^{\beta}=0, \\
\omega_{\alpha}^{A}(w) & =w_{\alpha}^{A}-F_{\alpha \beta}^{A} w^{\beta}-F_{\alpha B}^{A} w^{B}-F_{\alpha B}^{A \Gamma} w_{\Gamma}^{B}=0 .
\end{aligned}
$$

In Section 3 we have proved that (ii) implies (iii) in Theorem 1.1, i.e. we may assume that the compatibility conditions (1.4) and (1.5) hold. Now, a straightforward but tedious computation, using (3.8) to substitute in (5.17), and (5.14) and (5.19) to substitute in (5.18), shows that $w=w^{\alpha} e_{\alpha}$, which proves the desired inclusion $E \subset T_{z_{0}} S_{u^{A}}$.

\section{KäHLER ORDINARY AND KÄHLER REGULAR INTEGRAL ELEMENTS}

In this section, we recall the notions of Kähler ordinary and Kähler regular integral elements for an exterior differential system (see $\left[\mathrm{BCG}^{3} 91\right]$ ) and verify them for the ideal $\mathcal{I}$ introduced in the previous section. Let $t_{0} \in \mathbb{R}^{N}$ and $\mathcal{F}$ a family of germs at $t_{0}$ of real-analytic functions vanishing at $t_{0}$. Recall that $t_{0}$ is called an ordinary zero of $\mathcal{F}$ if there are $f_{1}, \ldots f_{\kappa} \in \mathcal{F}$ such that

$$
\operatorname{Rk}\left(\frac{\partial f_{i}}{\partial t^{j}}\left(t_{0}\right)\right)=\kappa
$$

where $i=1, \ldots, \kappa$, and $j=1, \ldots, N$, and the germ at $t_{0}$ of the real-analytic set $\{t: f(t)=$ $0, \forall f \in \mathcal{F}\}$ coincides with the germ at $t_{0}$ of $\left\{t: f_{1}(t)=\ldots=f_{\kappa}(t)=0\right\}$.

For a fixed $l \geq k$, we shall use the following convention. Lower-case $a, a^{\prime}, a^{\prime \prime}, \ldots$ run over the set of indices $\{1, \ldots, k, \ldots, l\}$, and $b, b^{\prime}, b^{\prime \prime}, \ldots$ run over the complementary set $\{l+1, \ldots, n\}$. (Note that if $l=k$, then the indices $a, a^{\prime} \ldots$ run over the same set as the capital Greek indices $\Lambda, \Gamma, \ldots$, whereas $b, b^{\prime}, \ldots$ run over the same set as lower-case Greek 
indices $\alpha, \beta, \ldots)$ Let $z_{0}:=\left(\left(x^{i}\right)_{0},\left(p^{A}\right)_{0},\left(p_{i}^{A}\right)_{0}\right)$ with $\left(p_{\alpha}^{A}\right)_{0}=F_{\alpha}^{A}\left(\left(x^{i}\right)_{0},\left(p^{B}\right)_{0},\left(p_{\Lambda}^{B}\right)_{0}\right)$ and assume that $E_{0} \subset T_{z_{0}} \mathbb{R}^{n+m+n m}$ is an l-dimensional integral element whose basis is of the form

$$
\frac{\partial}{\partial x^{a}}+\left(c_{a}^{A}\right)_{0} \frac{\partial}{\partial p^{A}}+\left(c_{a j}^{A}\right)_{0} \frac{\partial}{\partial p_{j}^{A}}, \quad a=1, \ldots, l .
$$

For $z:=\left(x^{i}, p^{A}, p_{i}^{A}\right)$, let $G_{l}\left(T_{z} \mathbb{R}^{n+m+n m}\right)$, for $k \leq l \leq n+m+n m$, denote the Grassmannian of all $l$-dimensional subspaces of $T_{z} \mathbb{R}^{n+m+n m}$. Note that $\left(z_{0}, E_{0}\right)$ is a point in the Grassmannian bundle

$$
X_{l}:=\left\{(z, E): z \in \mathbb{R}^{n+m+n m}, E \in G_{l}\left(T_{z} \mathbb{R}^{n+m+n m}\right)\right\} .
$$

For any $(z, E) \in X_{l}$, sufficiently close to $\left(z_{0}, E_{0}\right)$, there is a (unique) basis for $E$ of the form

$$
\tilde{e}_{a}:=\frac{\partial}{\partial x^{a}}+c_{a}^{b} \frac{\partial}{\partial x^{b}}+c_{a}^{A} \frac{\partial}{\partial p^{A}}+c_{a j}^{A} \frac{\partial}{\partial p_{j}^{A}}, \quad a=1, \ldots, l,
$$

where $\tilde{e}_{a} \in T_{z} \mathbb{R}^{n+m+n m}$ and $\left(z, c_{a}^{b}, c_{a}^{A}, c_{a j}^{A}\right)$ is close to $\left(z_{0}, 0,\left(c_{a}^{A}\right)_{0},\left(c_{a j}^{A}\right)_{0}\right)$. The map $(z, E) \mapsto\left(z, c_{a}^{b}, c_{a}^{A}, c_{a j}^{A}\right)$ forms a local coordinate system for $X_{l}$ near $\left(z_{0}, E_{0}\right)$. Consider the family $\mathcal{F}$ of germs at $\left(z_{0}, 0,\left(c_{a}^{A}\right)_{0},\left(c_{a j}^{A}\right)_{0}\right)$ of real-analytic functions given by

$$
\begin{aligned}
f_{\alpha}^{A} & :=p_{\alpha}^{A}-F_{\alpha}^{A}, \\
g_{a}^{A} & :=\eta^{A}\left(\tilde{e}_{a}\right)=c_{a}^{A}-p_{a}^{A}-p_{b}^{A} c_{a}^{b}, \\
g_{\alpha a}^{A} & :=\omega_{\alpha}^{A}\left(\tilde{e}_{a}\right)=c_{a \alpha}^{A}-F_{\alpha a}^{A}-F_{\alpha b}^{A} c_{a}^{b}-F_{\alpha B}^{A} c_{a}^{B}-F_{\alpha B}^{A \Gamma} c_{a \Gamma}^{B}, \\
h_{a a^{\prime}}^{A} & :=\Omega^{A}\left(\tilde{e}_{a}, \tilde{e}_{a^{\prime}}\right)=c_{a a^{\prime}}^{A}+c_{a b}^{A} c_{a^{\prime}}^{b}-\left(c_{a^{\prime} a}^{A}+c_{a^{\prime} b}^{A} c_{a}^{b}\right),
\end{aligned}
$$

where the $F_{\alpha}^{A}$ and their derivatives are evaluated at the point $\left(x^{i}, p^{B}, p_{\Lambda}^{B}\right)$, and $\eta^{a}, \omega_{\alpha}^{A}$, and $\Omega^{A}$ are the differential forms given by (5.5). Observe that, by definition, an $l$-dimensional subspace $E \subset T_{z} \mathbb{R}^{n+m+n m}$, with $(z, E)$ sufficiently close to $\left(z_{0}, E_{0}\right)$, is an integral element if and only if its local coordinate $\left(z, c_{a}^{b}, c_{a}^{A}, c_{a j}^{A}\right)$ is a zero of the family $\mathcal{F}$.

Recall (see $\mathrm{BCG}^{3} 91$ ) that the integral element $E_{0}$ of an exterior differential system is said to be Kähler ordinary if $\left(z_{0}, E_{0}\right)$ is an ordinary zero of the family of the functions of $(z, E)$ obtained by evaluating $l$-forms from the system on the basis of $E$ chosen as above. In fact, one can use any basis of $E$ depending on $(z, E)$ in a real-analytic fashion. Thus, in case of our system $\mathcal{I}$, the element $E_{0}$ is Kähler ordinary if $\left(z_{0}, E_{0}\right)$ is an ordinary zero of the family $\mathcal{F}$ given by $(6.5)$. Further recall that an integral element $E_{0}$ is said to be Kähler regular if it is Kähler ordinary and if the dimension of the polar spaces $H(E)$ (defined by (5.6)) is constant for all integral elements $E \subset T_{z} \mathbb{R}^{n+m+n m}$ (of dimension $l$ ) with $(z, E)$ sufficiently close to $\left(z_{0}, E_{0}\right)$. We have the following theorem, in which the notation introduced above is used. 
Theorem 6.1. Assume that property (ii) of Theorem 1.1 holds and let $l \geq k$. If $E \subset$ $T_{z} \mathbb{R}^{n+m+n m}$ is an an l-dimensional integral element with $(z, E)$ sufficiently close to $\left(z_{0}, E_{0}\right)$ in $X_{l}$, then $E$ is Kähler regular.

For the proof of Theorem 6.1, we shall need the following lemma, whose proof is elementary and left to the reader.

Lemma 6.2. Let $V_{i j}(s), b_{i}(s)$, for $i=1, \ldots, M$ and $j=1, \ldots, N_{2}$, be real-analytic functions in a neighborhood $W$ of $s_{0} \in \mathbb{R}^{N_{1}}$. Denote by $\mathcal{G}$ the collection of real-analytic functions in $W \times \mathbb{R}^{N_{2}}$, affine in $t=\left(t^{1}, \ldots, t^{N_{2}}\right)$, given by $L_{i}(s, t):=V_{i j}(s) t^{j}-b_{i}(s)$ for $i=1, \ldots, M$. Assume that all functions in $\mathcal{G}$ vanish at $\left(s_{0}, t_{0}\right)$ for some $t_{0} \in \mathbb{R}^{N_{2}}$ and let $A:=\left\{(s, t) \in W \times \mathbb{R}^{N_{2}}: f(s, t)=0, \forall f \in \mathcal{G}\right\}$. If there exists an integer $\kappa \geq 0$ such that, for all $s_{1} \in W, \operatorname{dim}\left\{t:\left(s_{1}, t\right) \in A\right\}=\kappa$, then $\left(s_{0}, t_{0}\right)$ is an ordinary zero of $\mathcal{G}$.

Proof of Theorem 6.1. If we can show that $E$ is Kähler ordinary, then it follows immediately from Corollary 5.5 and Proposition 5.6 that $E$ is Kähler regular. Moreover, by definition, if $E_{0}$ is Kähler ordinary, then $E$ is also Kähler ordinary when $(z, E)$ is sufficiently close to $\left(z_{0}, E_{0}\right)$. Thus, it suffices to show that $E_{0}$ is Kähler ordinary. For this, we shall make use of Lemma 6.2 with $\mathcal{G}$ being the family $\mathcal{F}$ given in the local coordinates $\left(z, c_{a}^{b}, c_{a}^{A}, c_{a j}^{A}\right)$ by (6.5). Recall that $z=\left(x^{i}, p^{A}, p_{i}^{A}\right)$. We split the local coordinates $\left(z, c_{a}^{b}, c_{a}^{A}, c_{a j}^{A}\right)$ into $(s, t)$, with $s=\left(x^{i}, p^{A}, p_{\Lambda}^{A}, c_{a}^{b}\right)$ and $t=\left(p_{\alpha}^{A}, c_{a}^{A}, c_{a j}^{A}\right)$, and take $\left(s_{0}, t_{0}\right)$ to be the local coordinates of the point $\left(z_{0}, E_{0}\right) \in X_{l}$. Observe that the functions in $\mathcal{F}$ are of the form required by Lemma 6.2, i.e. affine in $t$. Thus, to prove Theorem 6.1] it suffices to show that the dimension of the affine planes $\left\{t:\left(s_{1}, t\right) \in A\right\}$ is constant in $s_{1}$, for $s_{1}$ close to $s_{0}$. Let us fix $s_{1}=\left(\left(x^{i}\right)_{1},\left(p^{A}\right)_{1},\left(p_{\Lambda}^{A}\right)_{1}, c_{a}^{b}\right)$ close to $s_{0}$. As in the proof of Proposition 5.6. consider the Cauchy problem (2.1) with (2.2) satisfied, where $\left(\left(x^{i}\right)_{0},\left(p^{A}\right)_{0},\left(p_{\Lambda}^{A}\right)_{0}\right)$ is replaced by $\left(\left(x^{i}\right)_{1},\left(p^{A}\right)_{1},\left(p_{\Lambda}^{A}\right)_{1}\right)$. By assumption, this Cauchy problem has an approximate solution $u^{A}\left(x^{i}\right)$ at $x_{1}:=\left(x^{i}\right)_{1}$. By Proposition 3.1, the derivatives of $u^{A}$ at $x_{1}$ satisfy (3.8). The jet graph of $u^{A}$ is parametrized by the map $\Psi$ given by (5.16). A straightforward computation, as in the proof of Proposition 5.6, shows that

$$
\begin{aligned}
& e_{\Lambda}:=\Psi_{*}\left(\partial / \partial x^{\Lambda}\right)=\frac{\partial}{\partial x^{\Lambda}}+u_{\Lambda}^{A} \frac{\partial}{\partial p^{A}}+u_{\Lambda \Gamma}^{A} \frac{\partial}{\partial p_{\Gamma}^{A}}+\left(F_{\alpha \Lambda}^{A}+F_{\alpha B}^{A} u_{\Lambda}^{B}+F_{\alpha B}^{A \Gamma} u_{\Lambda \Gamma}^{B}\right) \frac{\partial}{\partial p_{\alpha}^{A}} \\
& e_{\alpha}:=\Psi_{*}\left(\partial / \partial x^{\alpha}\right)=\frac{\partial}{\partial x^{\alpha}}+u_{\alpha}^{A} \frac{\partial}{\partial p^{A}}+u_{\alpha \Lambda}^{A} \frac{\partial}{\partial p_{\Lambda}^{A}}+u_{\alpha \beta}^{A} \frac{\partial}{\partial p_{\beta}^{A}}
\end{aligned}
$$

where the $\partial / \partial x^{i}$ are tangent vectors at the point $x_{1} \in \mathbb{R}^{n}$. Recall that the coefficients $u_{i}^{A}$, $u_{i j}^{A}$ appearing in (6.6) are evaluated at $x_{1}$ and hence, in particular, $u_{\Lambda}^{A}=\left(p_{\Lambda}^{A}\right)_{1}$. Moreover, these coefficients satisfy (3.8), and hence we can think of them (and also then of the $e_{i}$ ) as functions of $a_{\Lambda \Gamma}^{A}$. Let $z_{1}$ denote the point

$$
\left(\left(x^{i}\right)_{1},\left(p^{A}\right)_{1},\left(p_{\Lambda}^{A}\right)_{1}, F_{\alpha}^{A}\left(\left(x^{i}\right)_{1},\left(p^{B}\right)_{1},\left(p_{\Gamma}^{B}\right)_{1}\right)\right) \in \mathbb{R}^{n+m+n m} .
$$


We observe that each $e_{i} \in T_{z_{1}} \mathbb{R}^{n+m+n m}$ in (6.6) is an affine function of $\left(a_{\Lambda \Gamma}^{A}\right) \in \mathbb{R}^{m k(k+1) / 2}$, i.e. $e_{i}=e_{i}\left(a_{\Lambda \Gamma}^{A}\right)$, and that the functions $e_{\Omega}=e_{\Omega}\left(a_{\Lambda \Gamma}^{A}\right)$ are injective. Consider the map sending $\left(a_{\Lambda \Gamma}^{A}\right) \in \mathbb{R}^{m k(k+1) / 2}$ to the point $\left(z_{1}, E\right) \in X_{l}$, where $E \subset T_{z_{1}} \mathbb{R}^{n+m+n m}$ is spanned by the $l$ vectors $\tilde{e}_{a}:=e_{a}+c_{a}^{b} e_{b}$. Observe that $E$ is a subspace of $T_{z_{1}} S_{u^{A}}$, where $S_{u^{A}}$ is the jet graph of the approximate solution $u^{A}$, and hence is an integral element of $\mathcal{I}$. Using the local coordinates $(s, t)$ on $X_{l}$ introduced above, the mapping $\left(a_{\Lambda \Gamma}^{A}\right) \mapsto\left(z_{1}, E\right)$ satisfies $s=s_{1}$ and its $t$-component, $t=t\left(a_{\Lambda \Gamma}^{A}\right)$, is affine. Moreover, as the reader can verify, the map $t=t\left(a_{\Lambda \Gamma}^{A}\right)$ is injective when the $c_{a}^{b}$ are sufficiently small. By Proposition 5.6, any l-dimensional integral element $E \subset T_{z_{1}} \mathbb{R}^{n+m+n m}$ with $\left(z_{1}, E\right)$ close to $\left(z_{0}, E_{0}\right)$, is contained in $T_{z_{1}} S_{u^{A}}$, where $S_{u^{A}}$ is the jet graph through $z_{1}$ of an approximate solution $u^{A}$ at $x_{1}$. Consequently, for each $s_{1}$ close to $s_{0}$, the space of all $l$-dimensional integral elements $(z, E)$ with coordinates $\left(s_{1}, t\right)$ is parametrized by the affine map $\left(a_{\Lambda, \Gamma}^{A}\right) \mapsto\left(s_{1}, t\left(a_{\Lambda \Gamma}^{A}\right)\right)$ and hence its dimension is $\kappa=m k(k+1) / 2$. By Lemma 6.2, $\left(s_{0}, t_{0}\right)$ is an ordinary zero for $\mathcal{F}$, which completes the proof of Proposition 6.1 .

\section{Proof of (ii) $\Longrightarrow$ (i) IN Theorem 1.1}

We note that it suffices to prove that the Cauchy problem (2.1) is solvable at $\left(\left(x^{i}\right)_{0},\left(p^{B}\right)_{0},\left(p_{\Lambda}^{B}\right)_{0}\right)$. We let $S_{0} \subset \mathbb{R}^{n+m+n m}$ be the submanifold through $z_{0}:=\left(\left(x^{i}\right)_{0},\left(p^{B}\right)_{0},\left(p_{\Lambda}^{B}\right)_{0},\left(p_{\alpha}^{A}\right)_{0}\right)$, with $\left(p_{\alpha}^{A}\right)_{0}:=F_{\alpha}^{A}\left(\left(x^{i}\right)_{0},\left(p^{B}\right)_{0},\left(p_{\Lambda}^{B}\right)_{0}\right)$, parametrized by $\Phi: V \rightarrow \mathbb{R}^{n+m+n m}$, where $V$ is a sufficiently small open neighborhood of $\left(x^{\Lambda}\right)_{0}$ in $\mathbb{R}^{k}$ and

$$
\Phi\left(x^{\Lambda}\right):=\left(\left(x^{\Lambda}, x_{0}^{\alpha}\right), a^{A}\left(x^{\Lambda}\right),\left(a_{\Gamma}^{A}\left(x^{\Lambda}\right), F_{\alpha}^{A}\left(\left(x^{\Lambda}, x_{0}^{\alpha}\right), a^{B}\left(x^{\Lambda}\right), a_{\Gamma}^{B}\left(x^{\Lambda}\right)\right)\right)\right),
$$

where $a^{A}\left(x^{\Lambda}\right)$ is the Cauchy data in (2.1) satisfying (2.2). Since the forms (5.3) vanish when restricted to $S_{0}$, the latter is an integral submanifold for the differential ideal $\mathcal{I}$ introduced in Section [5. Let $E_{0}$ be the tangent space $T_{z_{0}} S_{0}$, and observe that $E_{0}$ is an integral element of dimension $l=k$ with a basis of the form (6.2). By Theorem 6.1, the integral element $E:=T_{z} S_{0}$ is Kähler regular for every $z \in S_{0}$ with $z$ sufficiently close to $z_{0}$, and $\operatorname{dim} H(E)=n$ (by Corollary 5.5 and Proposition 5.6). Recall that an integral submanifold is called Kähler regular if its tangent space at every point is Kähler regular. In view of the above observation, $S_{0}$ is Kähler regular in some neighborhood of $z_{0}$. Let

$$
R_{1}:=\left\{\left(x^{i}, p^{A}, p_{i}^{A}\right) \in \mathbb{R}^{n+m+n m}: x^{k+2}=x_{0}^{k+2}, \ldots, x^{n}=x_{0}^{n}\right\} .
$$

Note that $R_{1}$ has codimension $r=n-k-1$ and is transverse to $H(E)$, for all $E=T_{z} S_{0}$ for $z \in S_{0}$ near $z_{0}$, by Corollary 5.5 and Proposition 5.6. (Indeed, $H(E)$ is a graph over $T_{x} \mathbb{R}^{n}$, being a tangent space of the jet graph of an approximate solution, and the variables $\left(p^{A}, p_{i}^{A}\right)$ are free in $R_{1}$.) By the Cartan-Kähler Theorem (Theorem 2.2 in [BCG $\left.{ }^{3} 91\right]$ ), there exists a real-analytic integral submanifold $S_{1}$ of dimension $l=k+1$ through $z_{0}$ such that $S_{0} \subset S_{1} \subset R_{1}$. By Proposition 5.6, the tangent space $T_{z_{0}} S_{1}$ is contained in $T_{z_{0}} S_{u^{A}}$ for the jet graph $S_{u^{A}}$ of some approximate solution $u^{A}\left(x^{i}\right)$ at $x_{0}$. Since the projection $\pi: \mathbb{R}^{n+m+n m} \rightarrow \mathbb{R}^{n}$ restricted to $S_{u^{A}}$ is a diffeomorphism, we conclude that the projection 
$\pi$ restricted to $S_{1}$ near $z_{0}$ is a diffeomorphism onto a $(k+1)$-dimensional submanifold through $x_{0}$ in $\mathbb{R}^{n}$. Since $S_{1}$ is also contained in $R_{1}$, we conclude that $T_{z_{0}} S_{1}$ has a basis of the form (6.2). Hence, it follows that $S_{1}$ is Kähler regular near $z_{0}$ by Theorem 6.1, and we have $\operatorname{dim} H\left(T_{z} S_{1}\right)=n$ for every $z \in S_{1}$ near $z_{0}$ (again by Corollary [5.5] and Proposition 5.6). Repeating the argument with $S_{0}$ replaced by $S_{1}$ and $R_{1}$ replaced by

$$
R_{2}:=\left\{\left(x^{i}, p^{A}, p_{i}^{A}\right) \in \mathbb{R}^{n+m+n m}: x^{k+3}=x_{0}^{k+3}, \ldots, x^{n}=x_{0}^{n}\right\},
$$

we obtain a real-analytic integral submanifold $S_{2}$ of dimension $l=k+2$ through $z_{0}$ such that $S_{0} \subset S_{1} \subset S_{2} \subset R_{2}$. Again, $T_{z_{0}} S_{2}$ has a basis of the form (6.2), $S_{2}$ is Kähler regular near $z_{0}$, and we have $\operatorname{dim} H\left(T_{z} S_{2}\right)=n$ for $z \in S_{2}$ near $z_{0}$. We continue this process, inductively producing integral submanifolds whose dimensions increase by one at each step. The process will end after $n-k$ steps with an $n$-dimensional real-analytic integral submanifold $S:=S_{n-k}$ through $z_{0}$ with the property that the projection $\pi: \mathbb{R}^{n+m+n m} \rightarrow$ $\mathbb{R}^{n}$ restricted to $S$ is a diffeomorphism, i.e. $d x_{1} \wedge \ldots \wedge d x_{n} \neq 0$ on $S$. As observed in the beginning of Section 5, this produces a real-analytic solution to the Cauchy problem (2.1). The uniqueness of the solution follows easily from a standard power series argument. The details are left to the reader. This completes the proof of the implication (ii) $\Longrightarrow$ (i) in Theorem 1.1.

\section{A system of Monge-Ampère type}

Let $u\left(x^{i}\right)$ be a real-valued function defined near the origin in $\mathbb{R}^{n}$. Denote by $H u\left(x^{i}\right)$ the Hessian of $u$, i.e. the symmetric $n \times n$-matrix of second order derivatives $\left(u_{i j}\right)_{i, j=1}^{n}$. As an application of Theorem 1.1, we shall give a construction of all real-analytic, real-valued functions $u$ defined near 0 in $\mathbb{R}^{n}$ such that the rank of $H u$ is identically one. We shall restrict our attention to those $u$ for which

$$
\delta(u):=u_{11}
$$

satisfies $\delta(u)(0) \neq 0$. Clearly every $u$ with nonvanishing $H u$ at 0 can be put into this form by a linear change of coordinates in $\mathbb{R}^{n}$. Recall, from the introduction, the differential operators $\Delta_{\alpha \beta}$ given by the $2 \times 2$-minors

$$
\Delta_{\alpha \beta}(u):=\operatorname{det}\left(\begin{array}{cc}
u_{11} & u_{1 \beta} \\
u_{\alpha 1} & u_{\alpha \beta}
\end{array}\right)
$$

for $\alpha, \beta \in\{2, \ldots, n\}$. (We will continue to use the notation and conventions established in previous sections with $k=1$.) Observe that $\Delta_{\alpha \beta}(u)=\Delta_{\beta \alpha}(u)$. The following statement is a consequence of a standard linear algebra argument:

Proposition 8.1. Let $u$ be a real-valued, real-analytic function defined near 0 in $\mathbb{R}^{n}$. Assume that $\delta(u)(0) \neq 0$. Then, the rank of $H u$ is identically one near 0 if and only if $\Delta_{\alpha \beta}(u)=0$ for all $\alpha, \beta \in\{2, \ldots, n\}$. 
To find all real-analytic $u$ such that the rank of $H u$ is identically one and $\delta(u)(0) \neq 0$, we shall consider the overdetermined Cauchy problem

$$
\left\{\begin{array}{l}
\Delta_{\alpha \beta}(u)=0, \\
u\left(x^{1}, 0\right)=a\left(x^{1}\right), \quad u_{\alpha}\left(x^{1}, 0\right)=a_{\alpha}\left(x^{1}\right), \quad \alpha, \beta=2, \ldots, n,
\end{array}\right.
$$

where the $n$ functions $a\left(x^{1}\right), a_{\alpha}\left(x^{1}\right)$ are real-valued and real-analytic near 0 in $\mathbb{R}$. In order to ensure that $\delta(u)(0) \neq 0$ we shall require $a^{\prime \prime}(0) \neq 0$. We shall in fact consider the more general Cauchy problem, as in Theorem 1.3 ,

$$
\left\{\begin{array}{l}
\Delta_{\alpha \beta}(u)=f_{\alpha \beta}\left(x^{j}, u_{11}\right), \\
u\left(x^{1}, x_{0}^{\alpha}\right)=a\left(x^{1}\right), \quad u_{\alpha}\left(x^{1}, x_{0}^{\alpha}\right)=a_{\alpha}\left(x^{1}\right), \quad \alpha, \beta=2, \ldots, n,
\end{array}\right.
$$

where the $f_{\alpha \beta}\left(x^{j}, t\right),\left(x^{j}\right)_{0}, a\left(x^{1}\right)$, and $a_{\alpha}\left(x^{1}\right)$ are as in Theorem 1.3. Naturally, we have to impose the symmetry condition $f_{\alpha \beta}=f_{\beta \alpha}$. We now give the proof of Theorem 1.3.

Proof of Theorem 1.3. We shall reduce the Cauchy problem (8.4) to a Cauchy problem for a system of first order partial differential equations of the form (2.1), and verify that the compatibility conditions of (iii) in Theorem 1.1 are equivalent to the $f_{\alpha \beta}$ being of the form $f_{\alpha \beta}\left(x^{j}, t\right)=g_{\alpha \beta}\left(x^{j}\right) t$ with $g_{\alpha \beta}$ satisfying (1.11). The conclusion of Theorem 1.3 then follows from Theorem 1.1. In the notation used in Theorem 1.1, we let $m=n+1$ and, for a real-valued $u$, we define $u^{A}, 1 \leq A \leq n+1$, as follows

$$
u^{i}:=u_{i}=\frac{\partial u}{\partial x^{i}}, 1 \leq i \leq n, \quad u^{n+1}:=u \text {. }
$$

We define, for a vector valued function $u^{i}$ with $1 \leq i \leq n$, the first order differential operators

$$
\Delta_{\alpha}^{\beta}\left(u^{i}\right):=\operatorname{det}\left(\begin{array}{ll}
u_{1}^{1} & u_{1}^{\beta} \\
u_{\alpha}^{1} & u_{\alpha}^{\beta}
\end{array}\right),
$$

for $\alpha, \beta \in\{2, \ldots, n\}$. By construction, a solution $u$ to the second order Cauchy problem (8.4) yields a solution $u^{A}$, via (8.5), to the first order Cauchy problem:

$$
\left\{\begin{array}{l}
u_{\alpha}^{1}=u_{1}^{\alpha}, \\
\Delta_{\alpha}^{\beta}\left(u^{i}\right)=f_{\alpha \beta}\left(x^{j}, u_{1}^{1}\right), \\
u_{\alpha}^{n+1}=u^{\alpha}, \\
u^{1}\left(x^{1}, x_{0}^{\alpha}\right)=a^{\prime}\left(x^{1}\right), u^{\alpha}\left(x^{1}, x_{0}^{\alpha}\right)=a_{\alpha}\left(x^{1}\right), \quad \alpha=2, \ldots, n . \\
u^{n+1}\left(x^{1}, x_{0}^{\alpha}\right)=a\left(x^{1}\right),
\end{array}\right.
$$

Note that we did not include the equation $u_{1}^{n+1}=u^{1}$ which does not contain a derivative transversal to the line $\left\{\left(x^{1}, x_{0}^{\alpha}\right)\right\}$. However, it actually follows from other equations. In fact, we have the converse: 
Lemma 8.2. If $u^{A}, 1 \leq A \leq n+1$, is a solution to the Cauchy problem (8.7), then $u:=u^{n+1}$ is a solution to the Cauchy problem (8.4) (as a germ at $\left.\left(x^{j}\right)_{0}\right)$.

Proof. Clearly, $u:=u^{n+1}$ satisfies the boundary condition (i.e. the last line) in (8.4). Thus, to prove the lemma, we must show that $\Delta_{\alpha \beta}(u)=f_{\alpha \beta}\left(x^{j}, u_{11}\right)$. To do this it suffices to show that

$$
\begin{aligned}
& u_{\alpha \beta}=u_{\beta}^{\alpha}, \\
& u_{1 \beta}=u_{1}^{\beta}, u_{\alpha 1}=u_{\alpha}^{1}, \\
& u_{11}=u_{1}^{1},
\end{aligned}
$$

since this implies $\Delta_{\alpha \beta}(u)=\Delta_{\alpha}^{\beta}\left(u^{i}\right)=f_{\alpha \beta}\left(x^{j}, u_{11}\right)$. The first two lines in (8.8) follow directly from the differential equations in (8.7) (using the symmetry of $u_{\alpha \beta}$ in $\alpha$ and $\beta$ ). To check the remaining identity in (8.8), we observe that the initial data conditions in (8.7) imply that it holds when $x^{\alpha}=x_{0}^{\alpha}$. On the other hand, by differentiating the first and third lines in (8.7), we obtain

$$
u_{\alpha 11}=u_{\alpha 11}^{n+1}=u_{11}^{\alpha}=u_{\alpha 1}^{1},
$$

and, hence, $\left(u_{11}-u_{1}^{1}\right)_{\alpha}=0$. Since $u_{11}=u_{1}^{1}$ when $x^{\alpha}=x_{0}^{\alpha}$, we conclude, by a uniqueness argument, that the identities in the last line of (8.8) hold. This completes the proof of the lemma.

We now observe that the Cauchy problem (8.7) decouples into the two Cauchy problems

$$
\left\{\begin{array}{l}
u_{\alpha}^{1}=u_{1}^{\alpha}, \\
\Delta_{\alpha}^{\beta}\left(u^{i}\right)=f_{\alpha}^{\beta}\left(x^{j}, u_{1}^{1}\right), \quad \alpha, \beta=2, \ldots, n, \\
u^{i}\left(x^{1}, x_{0}^{\alpha}\right)=a^{i}\left(x^{1}\right),
\end{array}\right.
$$

and

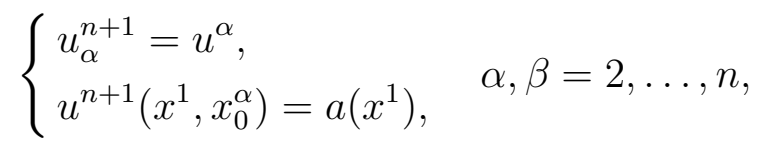

where the Cauchy data $a^{i}$ in (8.9) are taken to be $a^{1}:=a^{\prime}$ and $a^{\alpha}:=a_{\alpha}$, and the right hand side $f_{\alpha}^{\beta}:=f_{\alpha \beta}$.

Proposition 8.3. Let $f_{\alpha}^{\beta}\left(x^{i}, t\right)$, for $2 \leq \alpha, \beta \leq n$, with $n>2$, be real-analytic functions in a connected open subset $U \times V \subset \mathbb{R}^{n} \times(\mathbb{R} \backslash\{0\})$. Then the following two conditions are equivalent:

(i) For any choice of real-analytic Cauchy data $a^{i}\left(x^{1}\right)$ near $x_{0}$ in $U$ such that $\left(a^{1}\right)^{\prime}\left(x_{0}^{1}\right) \in V$, there exists a unique real-analytic solution $u^{j}\left(x^{i}\right)$ near $x_{0}$ to the Cauchy problem (8.9).

(ii) The functions $f_{\alpha}^{\beta}$ are of the form $f_{\alpha}^{\beta}\left(x^{i}, t\right)=g_{\alpha}^{\beta}\left(x^{i}\right) t$ with $g_{\alpha}^{\beta}$ satisfying

$$
g_{\alpha 1}^{\beta}=0, \quad g_{\alpha \beta}^{\gamma}=g_{\beta \alpha}^{\gamma}, \quad \alpha, \beta, \gamma=2, \ldots, n .
$$


Moreover, if $f_{\alpha}^{\beta}=f_{\beta}^{\alpha}$, then any solution $u^{j}\left(x^{i}\right)$ to (8.9) satisfies $u_{\beta}^{\alpha}=u_{\alpha}^{\beta}$ for $2 \leq \alpha, \beta \leq$ $n$.

Proof. Let us rewrite the differential equations in (8.9) explicitly in the form of (22.1):

$$
\left\{\begin{array}{l}
u_{\alpha}^{1}=u_{1}^{\alpha}, \\
u_{\alpha}^{\beta}=\frac{u_{1}^{\beta} u_{1}^{\alpha}+f_{\alpha}^{\beta}}{u_{1}^{1}}, \quad \alpha, \beta=2, \ldots, n .
\end{array}\right.
$$

In the second line, we have used the first line to replace $u_{\alpha}^{1}$ by $u_{1}^{\alpha}$. We remark that, in the notation of Theorem 1.1, we have here $m=n$ and $k=1$, so that the indices $A, B$, etc. run over the set $\{1, \ldots, n\}$ (i.e. the same index set as $i, j$, etc.) and $\Lambda, \Gamma$, etc. are all 1, and the right-hand side functions are given by

$$
F_{\alpha}^{1}\left(x^{i}, p^{B}, p_{1}^{B}\right)=p_{1}^{\alpha}, \quad F_{\alpha}^{\beta}\left(x^{i}, p^{B}, p_{1}^{B}\right)=\frac{p_{1}^{\beta} p_{1}^{\alpha}+f_{\alpha}^{\beta}\left(x^{i}, p_{1}^{1}\right)}{p_{1}^{1}} .
$$

We compute the derivatives of $F_{\alpha}^{A}$ that appear in (1.4) and (1.5) and that are not 0 :

$$
\left\{\begin{array}{l}
F_{\alpha i}^{\gamma}=\frac{f_{\alpha i}^{\gamma}\left(x^{j}, p_{1}^{1}\right)}{p_{1}^{1}} \\
F_{\alpha \alpha}^{11}=1 \\
F_{\alpha 1}^{\beta 1}=-\frac{p_{1}^{\alpha} p_{1}^{\beta}+f_{\alpha}^{\beta}}{\left(p_{1}^{1}\right)^{2}}+\frac{f_{\alpha 1}^{\beta 1}}{p_{1}^{1}} \\
F_{\alpha \gamma}^{\beta 1}=\frac{\delta_{\alpha \gamma} p_{1}^{\beta}+\delta_{\beta \gamma} p_{1}^{\alpha}}{p_{1}^{1}}
\end{array}\right.
$$

where $\delta_{\alpha \gamma}=\delta_{\alpha}^{\gamma}$ denotes the Kronecker delta symbol. We remark that the compatibility conditions (1.4) and (1.5) simply say that the functions in their left hand sides (or, equivalently, the right hand sides) are symmetric in $\alpha$ and $\beta$ for any $A \in\{1, \ldots, n\}$ in the case of (1.4), and for any $A, C \in\{1, \ldots, n\}$ and $\Gamma=\Lambda=1$ in the case of (1.5). We compute the left hand side of (1.4) with $A=1$ to obtain

$$
\Phi_{\alpha \beta}^{1}=0+0+F_{\alpha B}^{11}\left(F_{\beta 1}^{B}+0\right)=F_{\beta 1}^{\alpha}=\frac{f_{\beta 1}^{\alpha}}{p_{1}^{1}} .
$$

Next, we compute the left hand side of (1.4) with $A=\gamma$ :

$$
\Phi_{\alpha \beta}^{\gamma}=F_{\alpha \beta}^{\gamma}+0+F_{\alpha B}^{\gamma 1}\left(F_{\beta 1}^{B}+0\right)=\frac{f_{\alpha \beta}^{\gamma}}{p_{1}^{1}}+\frac{\delta_{\alpha \epsilon} p_{1}^{\gamma}+\delta_{\gamma \epsilon} p_{1}^{\alpha}}{\left(p_{1}^{1}\right)^{2}} f_{\beta 1}^{\epsilon}=\frac{f_{\alpha \beta}^{\gamma}}{p_{1}^{1}}+\frac{p_{1}^{\gamma} f_{\beta 1}^{\alpha}+p_{1}^{\alpha} f_{\beta 1}^{\gamma}}{\left(p_{1}^{1}\right)^{2}}
$$


We now compute the left hand side of (1.5) with $A=C=1$ :

$$
\Psi_{\alpha \beta 1}^{111}=2 F_{\alpha B}^{11} F_{\beta 1}^{B 1}=-2 \frac{p_{1}^{\alpha} p_{1}^{\beta}+f_{\alpha}^{\beta}}{\left(p_{1}^{1}\right)^{2}}+2 \frac{f_{\alpha 1}^{\beta 1}}{p_{1}^{1}} .
$$

The left hand side of (1.5) with $A=1, C=\gamma$ is given by

$$
\Psi_{\alpha \beta \gamma}^{111}=2 F_{\alpha B}^{11} F_{\beta \gamma}^{B 1}=2 \frac{\delta_{\alpha \gamma} p_{1}^{\beta}+\delta_{\beta \gamma} p_{1}^{\alpha}}{p_{1}^{1}},
$$

and with $A=\gamma, C=1$ by

$$
\begin{aligned}
\Psi_{\alpha \beta 1}^{\gamma 11}=2 F_{\alpha B}^{\gamma 1} F_{\beta 1}^{B 1} & =2\left(\delta_{\alpha \epsilon} p_{1}^{\gamma}+\delta_{\gamma \epsilon} p_{1}^{\alpha}\right)\left(-\frac{p_{1}^{\epsilon} p_{1}^{\beta}+f_{\beta}^{\epsilon}}{\left(p_{1}^{1}\right)^{3}}+\frac{f_{\beta 1}^{\epsilon 1}}{\left(p_{1}^{1}\right)^{2}}\right) \\
& =-2 \frac{2 p_{1}^{\alpha} p_{1}^{\beta} p_{1}^{\gamma}+p_{1}^{\gamma} f_{\beta}^{\alpha}+p_{1}^{\alpha} f_{\beta}^{\gamma}}{\left(p_{1}^{1}\right)^{3}}+2 \frac{p_{1}^{\gamma} f_{\beta 1}^{\alpha 1}+p_{1}^{\alpha} f_{\beta 1}^{\gamma 1}}{\left(p_{1}^{1}\right)^{2}}
\end{aligned}
$$

and finally with $A=\gamma$ and $C=\lambda$ by

$$
\begin{aligned}
\Psi_{\alpha \beta \lambda}^{\gamma 11}=2 F_{\alpha B}^{\gamma 1} F_{\beta \lambda}^{B 1} & =2 F_{\alpha 1}^{\gamma 1} F_{\beta \lambda}^{11}+2 F_{\alpha \epsilon}^{\gamma 1} F_{\beta \lambda}^{\epsilon 1} \\
& =2\left(-\frac{p_{1}^{\gamma} p_{1}^{\alpha}+f_{\alpha}^{\gamma}}{\left(p_{1}^{1}\right)^{2}}+\frac{f_{\alpha 1}^{\gamma 1}}{p_{1}^{1}}\right) \delta_{\beta \lambda}+2 \frac{\left(\delta_{\alpha \epsilon} p_{1}^{\gamma}+\delta_{\gamma \epsilon} p_{1}^{\alpha}\right)\left(\delta_{\beta \lambda} p_{1}^{\epsilon}+\delta_{\lambda \epsilon} p_{1}^{\beta}\right)}{\left(p_{1}^{1}\right)^{2}} \\
& =2 \frac{\delta_{\alpha \lambda} p_{1}^{\gamma} p_{1}^{\beta}+\delta_{\beta \lambda} p_{1}^{\alpha} p_{1}^{\gamma}+\delta_{\gamma \lambda} p_{1}^{\beta} p_{1}^{\alpha}}{\left(p_{1}^{1}\right)^{2}}-2\left(\frac{f_{\alpha}^{\gamma}}{\left(p_{1}^{1}\right)^{2}}-\frac{f_{\alpha 1}^{\gamma 1}}{p_{1}^{1}}\right) \delta_{\beta \lambda} .
\end{aligned}
$$

Since $n>2$, the expressions in (8.20) are symmetric in $\alpha$ and $\beta$ if and only if

$$
\frac{f_{\alpha}^{\gamma}}{\left(p_{1}^{1}\right)^{2}}-\frac{f_{\alpha 1}^{\gamma 1}}{p_{1}^{1}}=0, \quad \alpha, \gamma=2, \ldots, n,
$$

or, equivalently,

$$
f_{\alpha 1}^{\gamma 1}=\frac{f_{\alpha}^{\gamma}}{p_{1}^{1}}
$$

The latter is an ordinary differential equation (with parameters $x^{i}$ ) whose solutions are of the form

$$
f_{\alpha}^{\gamma}\left(x^{i}, t\right)=g_{\alpha}^{\gamma}\left(x^{i}\right) t .
$$

In view of (8.21), the expressions (8.15)-(8.20) become

$$
\begin{gathered}
\Phi_{\alpha \beta}^{1}=g_{\beta 1}^{\alpha}, \quad \Phi_{\alpha \beta}^{\gamma}=g_{\alpha \beta}^{\gamma}+\frac{p_{1}^{\gamma} g_{\beta 1}^{\alpha}+p_{1}^{\alpha} g_{\beta 1}^{\gamma}}{p_{1}^{1}} \\
\Psi_{\alpha \beta 1}^{111}=-2 \frac{p_{1}^{\alpha} p_{1}^{\beta}}{\left(p_{1}^{1}\right)^{2}}, \quad \Psi_{\alpha \beta \gamma}^{111}=2 \frac{\delta_{\alpha \gamma} p_{1}^{\beta}+\delta_{\beta \gamma} p_{1}^{\alpha}}{p_{1}^{1}}, \quad \Psi_{\alpha \beta 1}^{\gamma 11}=-4 \frac{p_{1}^{\alpha} p_{1}^{\beta} p_{1}^{\gamma}}{\left(p_{1}^{1}\right)^{3}},
\end{gathered}
$$




$$
\Psi_{\alpha \beta \lambda}^{\gamma 11}=2 \frac{\delta_{\alpha \lambda} p_{1}^{\gamma} p_{1}^{\beta}+\delta_{\beta \lambda} p_{1}^{\alpha} p_{1}^{\gamma}+\delta_{\gamma \lambda} p_{1}^{\beta} p_{1}^{\alpha}}{\left(p_{1}^{1}\right)^{2}}
$$

We note that all the expressions (8.23)-(8.24) are symmetric in $\alpha$ and $\beta$. If we take $p_{1}^{\gamma}=p_{1}^{\alpha}=0$ in the second expression in (8.22), we see that this expression is symmetric in $\alpha$ and $\beta$ if and only if $g_{\alpha \beta}^{\gamma}$ is. Now, we take $p_{1}^{\alpha}=0$ and $p_{1}^{\gamma}=1$ in the second expression in (8.22). Since $g_{\alpha \beta}^{\gamma}$ must be symmetric in $\alpha$, $\beta$, we conclude that $g_{\beta 1}^{\alpha}$ must be symmetric in $\alpha, \beta$. Finally, since $g_{\alpha \beta}^{\gamma}$ and $g_{\beta 1}^{\alpha}$ must be symmetric in $\alpha, \beta$, we conclude that $p_{1}^{\alpha} g_{\beta 1}^{\gamma}$ must also be symmetric in $\alpha, \beta$. However, since $g_{\beta 1}^{\gamma}$ is a function of $x^{i}$ alone, this can only happen if $g_{\beta 1}^{\gamma}=0$ (where we again use $n>2$ ). Hence, the implication (i) $\Longrightarrow$ (ii) follows from Theorem 1.1. Moreover, if the conditions in (ii) are satisfied, then all the expressions (8.15)-(8.20) are symmetric in $\alpha, \beta$ and, hence, the implication (ii) $\Longrightarrow$ (i) also follows from Theorem 1.1. If $f_{\beta}^{\alpha}=f_{\alpha}^{\beta}$, then the symmetry $u_{\beta}^{\alpha}=u_{\alpha}^{\beta}$ is immediate from the second line in (8.12).

We now complete the proof of Theorem 1.3 . To prove $(\mathrm{i}) \Longrightarrow$ (ii), we first note that the symmetry $f_{\alpha \beta}=f_{\beta \alpha}$ obviously follows from (1.10). We consider the first order Cauchy problem (8.9) with $f_{\alpha}^{\beta}:=f_{\alpha \beta}$. Assume (i) in Theorem 1.3. We claim that (i) of Proposition 8.3 holds. Let $a^{j}\left(x^{1}\right)$ be real-analytic Cauchy data near $x_{0}$ in $\mathbb{R}$ with $\left(a^{1}\right)^{\prime}\left(x_{0}\right) \neq 0$, and let $a\left(x^{1}\right)$ be a real-analytic function near $x_{0}$ such that $a^{\prime}\left(x^{1}\right)=a^{1}\left(x^{1}\right)$. Note that $a^{\prime \prime}\left(x_{0}\right)=\left(a^{1}\right)^{\prime}\left(x_{0}\right) \neq 0$. We now let $u\left(x^{i}\right)$ be the solution to the Cauchy problem (1.10) with Cauchy data $a\left(x^{1}\right)$ and $a_{\alpha}\left(x^{1}\right):=a^{\alpha}\left(x^{1}\right)$ (that exists in view of our assumption). The gradient $u^{j}\left(x^{i}\right):=u_{j}\left(x^{i}\right)$ solves the Cauchy problem (8.9). Hence, by Proposition 8.3, the conditions in (1.11) must also hold.

To prove the converse (ii) $\Longrightarrow(\mathrm{i})$, we let $a\left(x^{1}\right)$ and $a_{\alpha}\left(x^{1}\right)$ be real-analytic Cauchy data near $x_{0}$ in $U$ such that $a^{\prime \prime}\left(x_{0}^{1}\right) \in V$. We define $a^{1}\left(x^{1}\right):=a^{\prime}\left(x^{1}\right)$ and $a^{\alpha}\left(x^{1}\right):=a_{\alpha}\left(x^{1}\right)$ and note that $\left(a^{1}\right)^{\prime}\left(x_{0}\right) \neq 0$. We consider the Cauchy problem (8.9) with $f_{\alpha}^{\beta}:=f_{\alpha \beta}$ and Cauchy data $a^{j}\left(x^{1}\right)$ defined above. The conditions in (1.11) clearly imply those in (8.11). Hence, by Proposition 8.3, the Cauchy problem (8.9) has a real-analytic solution $u^{j}\left(x^{i}\right)$ and since $f_{\alpha}^{\beta}=f_{\beta}^{\alpha}$, we have $u_{\beta}^{\alpha}=u_{\alpha}^{\beta}$. Hence, the Cauchy problem (8.10), with data $a\left(x^{1}\right)$ as above, has a solution $u:=u^{n+1}$. By Lemma 8.2, the real-analytic function $u\left(x^{i}\right)$ is the solution to (8.4). This completes the proof of Theorem 1.3.

\section{Proof of Theorem 1.6}

In this section, we shall give the proof of Theorem 1.6 stated in the introducion. We retain the notation established in previous sections. We shall use $x$ as local coordinates on $\Sigma$ near 0 . We choose as a normal to $\Sigma$ at a point $(x, u(x))$ the vector

$$
N(x):=\left(u_{i}(x),-1\right)
$$


and, hence, the Gauss map $G: \Sigma \rightarrow S^{n} \subset \mathbb{R}^{n+1}$ is given by

$$
G(x)=\frac{N(x)}{|N(x)|} \text {. }
$$

Consider the mapping $P: S^{n} \cap\left\{(x, y) \in \mathbb{R}^{n+1}: y<0\right\} \rightarrow \mathbb{R}^{n}$ given by

$$
P\left(x,-\sqrt{1-|x|^{2}}\right):=\frac{x}{\sqrt{1-|x|^{2}}} .
$$

Note that the mapping $\left(x,-\sqrt{1-|x|^{2}}\right) \mapsto(P(x),-1)$ is the stereographic projection from the origin to the hyperplane $\left\{(x, y) \in \mathbb{R}^{n+1}: y=-1\right\}$. Consequently, we conclude that $P$ is a diffeomorphism and we have the identity $P \circ G=\left(u_{i}\right)$. Let $\Gamma:(-\epsilon, \epsilon) \rightarrow \mathbb{R}^{n}$, for $\epsilon>0$ sufficiently small, be the real-analytic curve $\Gamma:=P \circ\left(\left.\gamma\right|_{(-\epsilon, \epsilon)}\right)$ through the origin in $\mathbb{R}^{n}$. By assumption, $\Gamma$ is of the form

$$
\Gamma(t)=\left(\varphi(t), a_{2}(t), \ldots, a_{n}(t)\right),
$$

with $\varphi(0)=0, \varphi^{\prime}(0)=1, a_{\alpha}^{\prime}(0)=0$. We define $a\left(x^{1}\right)$ by

$$
a\left(x^{1}\right):=\int_{0}^{x^{1}} \varphi(t) d t .
$$

Note that $a^{\prime \prime}(0)=1 \neq 0$. Let $u(x)$ be the unique real-analytic solution to the Cauchy problem (8.4), with $f_{\alpha \beta} \equiv 0$ and $x_{0}=0$, given by Theorem 1.3 . Now, by construction, $u_{x}\left(x^{1}, 0\right)=\Gamma\left(x^{1}\right)$. Moreover, the Jacobian matrix of the gradient map $x \mapsto u_{x}(x)$ equals the Hessian of $u(x)$ and hence, by Proposition 8.1, the rank of this Jacobian matrix is one near 0 . This completes the proof of Theorem 1.6 .

\section{Proof of Proposition 1.7}

In this section, we give the proof of Proposition 1.7. Let $\Sigma \subset \mathbb{R}^{n+1}$ satisfy the assumptions in the proposition. We shall prove that there is an open set in the tube hypersurface $M:=\Sigma+i \mathbb{R}^{n+1}$ on which $M$ is $n$-nondegenerate. The fact that $M$ is holomorphically nondegenerate then follows from the connectedness of $M$ and a general result relating finite nondegeneracy and holomorphic nondegeneracy (see e.g. Theorem 11.5.1 in [BER99]). Moreover, it follows from the real-analyticity of the vector valued functions in (1.12), as $p$ varies over $M$, that $M$ is $n$-nondegenerate on a dense open set. The fact that $\ell(M)=n$ now follows from the lower semicontinuity of the rank of a collection of vector valued functions. Finally, the fact that the rank of the Levi form is one on a dense open set follows easily from the fact that the rank $r$ of the Levi form at $p \in M$ equals $R-1$, where $R$ denotes the rank of the collection of vectors in (1.12) at $p$ with $j=1$ and the fact that, on a dense open set, the rank of these vectors has to strictly increase with $j$.

After a rotation and translation in $\mathbb{R}^{n+1}$ if necessary, we may assume that $\Sigma$ is given as a graph through 0 of the form $y=u(x)$. Since the Gauss image $G(\Sigma)$ is assumed to be a curve, we may also assume that the rank of the Gauss map, or equivalently the rank of the 
Hessian of the graphing function $u(x)$ (see the discussion in the previous section), equals one in a neighborhood of 0 , and that $u_{11}(0) \neq 0$. Now, the tube hypersurface $M$ is then defined in a tube neighborhood $U+i \mathbb{R}^{n+1}$, for some open neighborhood $0 \in U \subset \mathbb{R}^{n+1}$, by an equation of the form $\operatorname{Re} w=u(\operatorname{Re} z)$, where $(z, w)$ are coordinates in $\mathbb{C}^{n} \times \mathbb{C}$ such that $\operatorname{Re} w=y$ and $\operatorname{Re} z=x$. We can also write the equation of $M$ in the form

$$
w=-\bar{w}+2 i u((z+\bar{z}) / 2) .
$$

If we write $q(z, \bar{z}):=2 i u((z+\bar{z}) / 2)$, then it is well known (see e.g. Corollary 11.2.14 in [BER99]) that $M$ is $l$-nondegenerate at at point $(z, w) \in M$ if and only if the collection of vectors

$$
q_{\bar{z} z^{I}}(z, \bar{z}), \quad I \in \mathbb{Z}_{+}^{n},|I| \leq j,
$$

spans $\mathbb{C}^{n}$ with $j=l$ and $l$ is the smallest integer with this property; we use here the notation $q_{\bar{z}}$ for the vector given by the anti-holomorphic gradient $q_{\bar{z}}:=\left(q_{\bar{z}^{i}}\right) \in \mathbb{C}^{n}$, and also multi-index notation as in (1.12), so that $z^{I}:=\left(z^{1}\right)^{I_{1}} \ldots\left(z^{n}\right)^{I_{n}}$. Thus, it follows from (10.2) that $M$ is $l$-nondegenerate along the fiber $(x, y)+i \mathbb{R}^{n+1}$, with $(x, y) \in \Sigma$, if and only if the collection of vectors

$$
u_{x x^{I}}(x), \quad I \in \mathbb{Z}_{+}^{n},|I| \leq j,
$$

spans $\mathbb{R}^{n}$ with $j=l$ and $l$ is the smallest integer with this property. We have the following lemma.

Lemma 10.1. Let $\Sigma$ be given by $y=u(x)$. The Gauss image $G(\Sigma)$ is not contained in a hyperplane if and only if the vectors in (10.3) evaluated at $x=0$ span $\mathbb{R}^{n}$ for some integer $j$.

Proof. Let $P: S^{n} \cap\left\{(x, y) \in \mathbb{R}^{n+1}: y<0\right\} \rightarrow \mathbb{R}^{n}$ denote the stereographic projection introduced in (9.3) and recall that $u_{x}(x)=(P \circ G)(x)$. It is easy to see that $G(\Sigma)$ is not contained in a hyperplane in $\mathbb{R}^{n+1}$ if and only if $P(G(\Sigma))$ is not contained in a hyperplane in $\mathbb{R}^{n}$. Moreover, $P(G(\Sigma))$ is contained in hyperplane if and only if there is a non-zero vector $a=\left(a^{i}\right) \in \mathbb{R}^{n}$ such that $a^{i} u_{x^{i}}(x)=0$ for all $x$ in a neighborhood of 0 . Since $u$ is real-analytic, the latter is equivalent to $a^{i} u_{x^{i} x^{I}}(0)=0$ for all multi-indices $I \in \mathbb{Z}_{+}^{n}$, which clearly is equivalent to the vectors in (10.3) evaluated at $x=0$ not spanning $\mathbb{R}^{n}$ for any integer $j$. This completes the proof of the lemma.

Since the rank of the Hessian of $u$ is one near 0 and $u_{11}(0) \neq 0$, we conclude by Proposition 8.1 that $u$ satisfies near 0 the differential equations $\Delta_{\alpha \beta}(u)=0$, where $\Delta_{\alpha \beta}$ are given by (8.2). Thus, for any $\alpha \in\{2, \ldots, n\}$,

$$
u_{x \alpha}=\frac{u_{1 \alpha}}{u_{11}} u_{x 1}
$$

where $u_{x i}=\left(u_{1 i}, \ldots, u_{n i}\right)$. An induction, left to the reader, using the identity (10.4) proves the following lemma. 
Lemma 10.2. Let $u(x)$ be a function such that the Hessian Hu has rank one at every point near 0 and $u_{11}(0) \neq 0$. Then, for any multi-index $I \in \mathbb{Z}_{+}^{n}$, the vector $u_{x x^{I}}(x)$ belongs to the span of the vectors

$$
u_{x\left(x^{1}\right)^{m}}(x), \quad m \leq|I| .
$$

Thus, we conclude, by Lemma 10.1 and 10.2 that, for some integer $j$, the collection of vectors $u_{x\left(x^{1}\right)^{m}}(0)$, with $m \leq j$, spans $\mathbb{R}^{n}$. It is easily seen that this is impossible unless the collection of vectors $u_{x\left(x^{1}\right)^{m}}(x)$, with $m \leq n$, spans $\mathbb{R}^{n}$ for all $x$ in a dense open set. In view of Lemma 10.2 and the remarks preceding Lemma 10.1, this proves that $M$ is $n$-nondegenerate on an open set and, hence, $\ell(M)=n$ as claimed. This completes the proof of Proposition 1.7 .

We conclude this paper with two remarks.

Remark 10.3. As consequence of Lemma 10.1 and the discussion preceding it we conclude that for a connected real-analytic tube hypersurface $M=\Sigma+i \mathbb{R}^{n+1}$, the following are equivalent:

(1) $M$ is holomorphically nondegenerate;

(2) $M$ is finitely nondegenerate at each point;

(3) the Gauss image $G(\Sigma)$ is not contained in a hyperplane of $\mathbb{R}^{n+1}$.

Note that, for a general connected real-analytic hypersurface of $\mathbb{C}^{n+1},(2)$ implies (1) but not vice versa.

Remark 10.4. It follows from the results in the last three sections (8, 9, and 10) that, for any $n$ real-analytic functions $a\left(x^{1}\right)$ and $a_{\alpha}\left(x^{1}\right)$ near 0 in $\mathbb{R}$ such that $a_{11}(0) \neq 0$, there is a unique tube hypersurface $M$ of the form Re $w=u(\operatorname{Re} z)$ such that the rank of the Levi form is one at every point near 0 and such that $u\left(x^{1}, 0\right)=a\left(x^{1}\right)$ and $u_{\alpha}\left(x^{1}, 0\right)=a_{\alpha}\left(x^{1}\right)$. The tube hypersurface will be finitely nondegenerate at 0 , and hence holomorphically nondegenerate, if the collection of vectors

$$
\left(a^{(m)}(0), a_{\alpha}^{(m-1)}(0)\right), \quad m=1,2, \ldots,
$$

spans $\mathbb{R}^{n}$.

\section{REFERENCES}

[AG04] Akivis, M. A.; Goldberg, V. V.: Differential Geometry of Varities with Degenerate Gauss Maps. CMS Books in Mathematics, Springer-Verlag, New York, NY, 2004.

[BER99] Baouendi, M. S.; Ebenfelt, P.; Rothschild, L. P.: Real submanifolds in complex space and their mappings. Princeton Mathematical Series, 47. Princeton University Press, Princeton, NJ, 1999. xii+404 pp.

[BHR96] Baouendi, M. S.; Huang, Xiaojun; Preiss Rothschild, L. P.: Regularity of CR mappings between algebraic hypersurfaces. Invent. Math., 125, (1996), no. 1, 13-36.

[BCG ${ }^{3}$ 91] Bryant, R. L.; Chern, S. S.; Gardner, R. B.; Goldschmidt, H. L.; Griffiths, P. A.: Exterior differential systems. Mathematical Sciences Research Institute Publications, 18. Springer-Verlag, New York, 1991. viii+475 pp. 
[C31] Cartan, E.: Sur la thorie des systmes en involution et ses applications la relativit. (French) Bull. Soc. Math. France, 59, (1931), 88-118.

[E01] Ebenfelt, P.: Uniformly Levi degenerate CR manifolds: the 5-dimensional case. Duke Math. J., 110, (2001), no. 1, 37-80. Erratum: Duke Math. J., 131, (2006), no. 3, 589-591.

[F07] Fels, G.: Locally homogeneous finitely nondegenerate CR-manifolds. Math. Res. Lett., 14, (2007), no. 6, 893-922.

[FK06] Fels, G.; Kaup, W.: Classification of Levi degenerate homogeneous CR-manifolds in dimension 5. Acta Math (to appear). http://arxiv.org/ps/math.CV/0610375

[FK07] Fels, G.; Kaup, W.: CR-manifolds of dimension 5: a Lie algebra approach. J. Reine Angew. Math., 604 (2007), 47-71.

[F77] Freeman, M.: Local biholomorphic straightening of real submanifolds. Ann. Math., (2), 106, (1977), no. 2, 319-352.

[G67] Goldschmidt, H.: Integrability criteria for systems of nonlinear partial differential equations. J. Differential Geometry, 1, (1967), 269-307.

[HN59] Hartman, P.; Nirenberg, L.: On spherical image maps whose Jacobians do not change sign. Amer. J. Math., 81, (1959), 901-920.

[R10] Riquier, C.: Les systèmes d'équations aux dérivées partielles., Gauthiers-Villars, Paris, 1910.

[S60] Sacksteder, R.: On hypersurfaces with no negative sectional curvatures. Amer. J. Math., 82, (1960), 609-630.

[T34] Thomas, J. M.: Riquier's existence theorems. Ann. of Math. (2), 35, (1934), no. 2, 306-311.

[W95] Wu, H.: Complete developable submanifolds in real and complex Euclidean spaces. Internat. J. Math., 6, (1995), no. 3, 461-489.

M. S. Baouendi, P. Ebenfelt: Department of Mathematics, University of California at San Diego, La Jolla, CA 92093-0112, USA

E-mail address: sbaouendi@ucsd.edu, pebenfel@math.ucsd.edu

D. Zaitsev: School of Mathematics, Trinity College, Dublin 2, Ireland

E-mail address: zaitsev@maths.tcd.ie 